\title{
ON THE IRREDUCIBILITY OF LOCALLY METRIC CONNECTIONS
}

\author{
FLORIN BELGUN, ANDREI MOROIANU
}

\begin{abstract}
A locally metric connection on a smooth manifold $M$ is a torsion-free connection $D$ on $T M$ with compact restricted holonomy group $\operatorname{Hol}_{0}(D)$. If the holonomy representation of such a connection is irreducible, then $D$ preserves a conformal structure on $M$. Under some natural geometric assumption on the life-time of incomplete geodesics, we prove that conversely, a locally metric connection $D$ preserving a conformal structure on a compact manifold $M$ has irreducible holonomy representation, unless $\operatorname{Hol}_{0}(D)=0$ or $D$ is the Levi-Civita connection of a Riemannian metric on $M$. This result generalizes Gallot's theorem on the irreducibility of Riemannian cones to a much wider class of connections. As an application, we give the geometric description of compact conformal manifolds carrying a tame closed Weyl connection with non-generic holonomy.
\end{abstract}

2010 Mathematics Subject Classification: Primary 53A30, 53C05, 53C29.

Keywords: closed Weyl connections, reducible holonomy, Riemannian cones.

\section{INTRODUCTION}

The restricted holonomy group of the Levi-Civita connection of a Riemannian manifold $(M, g)$ is (conjugate to) a closed subgroup of $\mathrm{SO}(n)$, and thus compact. This property actually characterizes locally the Levi-Civita connections: If $D$ is a torsion-free connection on some manifold $M$ with compact restricted holonomy group $\mathrm{Hol}_{0}(D)$, then every point $x$ of $M$ has a neighborhood with a Riemannian metric on it whose Levi-Civita connection is $D$. This explains the following:

Definition 1.1. A locally metric connection on a smooth manifold $M$ is a torsion-free connection $D$ on $T M$ with compact restricted holonomy group $\operatorname{Hol}_{0}(D)$.

A typical example of locally (but not globally) metric connection is the following:

Example 1.2. Let $\left(N, g^{N}\right)$ be a Riemannian manifold, $\lambda>1$ a real number and consider the product $\bar{N}:=\mathbb{R}_{+}^{*} \times N$. The group $\Gamma$ generated by the dilation $\gamma: \bar{N} \rightarrow \bar{N}, \gamma(t, x)=(\lambda t, x)$ consists of strict homotheties of the cone metric $\bar{g}:=d t^{2}+t^{2} g^{N}$ on $\bar{N}$, so the Levi-Civita connection of $\bar{g}$ induces a locally metric connection $D$ on $M:=\bar{N} / \Gamma=S^{1} \times N$ which is not globally metric (see also Example 2.5 below).

In this paper we study the reducibility question for locally metric connections. Let us first remark that an irreducible locally metric connection $D$ always preserves a conformal structure on $M$. Indeed, the lift $\tilde{D}$ of $D$ to the universal cover $\tilde{M}$ of $M$ has compact holonomy group $\operatorname{Hol}(\tilde{D})=\operatorname{Hol}_{0}(D)$, thus is the Levi-Civita connection of a Riemannian metric $\tilde{g}$ on $\tilde{M}$. Moreover, the fundamental group $\Gamma$ of $M$ acts on $\tilde{M}$ by $\tilde{D}$-affine transformations, hence by homotheties (for every $\gamma \in \Gamma$ the metric $\gamma^{*} g$ is $\tilde{D}$-parallel, thus homothetic to $g$ by the irreducibility hypothesis).

This work was partially supported by the ANR-10-BLAN 0105 grant of the Agence Nationale de la Recherche. 
Consequently, the conformal structure $[\tilde{g}]$ defined by $\tilde{g}$ is $\Gamma$-invariant, so it defines a $D$-parallel conformal structure on $M$.

Conversely, one can ask whether a locally metric connection $D$ preserving a conformal structure on $M$ is necessarily irreducible. The answer is negative in general, as shown by the following examples:

(a) $D$ is the Levi-Civita connection of a product of Riemannian manifolds;

(b) $M$ is the quotient of $\mathbb{R}^{n} \backslash\{0\}$ by the group $\Gamma$ generated by multiplication with some $\lambda>1$ and $D$ is the connection induced on $\left(\mathbb{R}^{n} \backslash\{0\}\right) / \Gamma$ by the Levi-Civita connection of the flat metric of $\mathbb{R}^{n} \backslash\{0\}$;

(c) $D$ is obtained like in Example 1.2 from the product of two Riemannian cones (which is itself a Riemannian cone, see [13]).

Remark now that in case (a) $D$ is globally metric, in case (b) $D$ is flat, whereas in case (c) the manifold $M$ is non-compact. It turns out that all known examples of reducible locally metric connections preserving a conformal structure fall in one of the three cases above. It is therefore tempting to make the following:

Conjecture 1.3. A locally metric conformal connection on a compact manifold $M$ which is neither flat, nor globally metric, has irreducible holonomy.

In spite of its simplicity, this statement is still open in full generality. Some evidence is provided by the beautiful theorem of Gallot [6] concerning the irreducibility of Riemannian cone metrics, which is the core of Bär's geometric description of compact manifolds with Killing spinors [1]. Indeed, Gallot's theorem, which says that if $(N, g)$ is compact then the Riemannian cone $(\bar{N}, \bar{g})$ is irreducible or flat, can be restated as follows: the locally metric connection $D$ on the compact manifold $M:=S^{1} \times N$ defined in Example 1.2 is either flat or irreducible (note that, by construction, $D$ is not globally metric).

One of the main results of this paper is the following:

Theorem 1.4. Conjecture 1.3 holds provided that D is tame (cf. Definition 3.2).

The tameness condition is related to the life-time of incomplete geodesics of the connection. It is satisfied by the connections covered by Gallot's theorem, by every connection $C^{1}$-close to them (cf. Theorem 1.6 and Remark 5.2), and by large classes of locally metric connections defined on generalized cylinders ( $c f$. Example 5.7).

We will now give a more detailed account of our results from the point of view of conformal geometry. On a conformal manifold $(M, c)$, the rôle of the Levi-Civita connection is played by the family of Weyl connections, which are torsion-free connections on the tangent bundle TM preserving the conformal structure [16]. Weyl connections can be either closed, or exact, (i.e. locally, resp. globally equal to the Levi-Civita connection of some Riemannian metric in the conformal class), or non-closed. In this framework, a locally metric connection compatible with a conformal structure $c$ is nothing but a closed Weyl connection on $(M, c)$.

As a consequence of the Merkulov-Schwachhöfer classification of groups occurring as holonomy of torsion-free connections [12], the holonomy group of every non-closed irreducible Weyl connection is the full conformal group in dimensions other than 4. In [3] we show that the reducible case is very interesting and, so far, little understood: The holonomy reduction defines locally a conformal 
product structure, and the holonomy group, although included in a product group, is not necessarily a product itself. In short, the restricted holonomy of a non-closed Weyl connection is either trivial, the full conformal group, some special groups in dimension 4, or it is reducible (in which case no complete description exists yet).

In contrast to that, the restricted holonomy of a closed Weyl connection is always a Riemannian holonomy (see Remark 2.4 below). However, not every Riemannian holonomy group occurs as holonomy of a closed, non-exact Weyl connection. More precisely, we show in Section 6.1 that the locally symmetric case and the quaternion Kähler holonomy $\mathrm{Sp}_{k} \cdot \mathrm{Sp}_{1}$ do not occur, while all other irreducible holonomy groups in the Berger list occur as restricted holonomy groups of closed, non-exact Weyl connections. Moreover, one can even realize them on compact manifolds, by means of the cone construction, $c f$. Theorem 6.1 for details.

Theorem 1.4 excludes (under the tameness assumption), the existence of non-trivial reducible holonomy groups of closed, non-exact, Weyl connection on a compact conformal manifold. Of course, an analogous statement can not hold for exact or non-closed Weyl connections. Simple counter-examples are Riemannian products for the first case and conformal products (see [3]) for the second one.

We now describe the strategy of the proof of the main results. To every closed, non-exact Weyl connection $D$ on a conformal manifold $(M, c)$, we associate its minimal Riemannian cover $\left(M_{0}, g_{0}\right)$, with the property that the deck transformation group acts on $M_{0}$ by strict homotheties, and the pull-back of $D$ to $M_{0}$ is the Levi-Civita connection of $g_{0}$. We obtain in this way a one-toone correspondence between closed, non-exact, Weyl connections on compact conformal manifolds and incomplete Riemannian manifolds carrying a co-compact group $\Gamma$ of strict homotheties acting freely and properly discontinuously, called cone-like manifolds (see also Remark 2.4 for an equivalent definition). Every Riemannian cone over a compact manifold is a cone-like manifold (see Example 2.5). However, while the group of strict homotheties is at least 1-dimensional on every a Riemannian cone, it is only a discrete group on general cone-like manifolds.

Our first result which is also of independent interest, gives an information (essential for the proof of Theorem 1.4) about the metric space structure of cone-like manifolds.

Theorem 1.5. Let $\left(M_{0}, g_{0}, \Gamma\right)$ be a cone-like space and let d denote the distance on $M$ induced by $g_{0}$. Then the metric completion of $\left(M_{0}, d\right)$ is a metric space $\widehat{M}_{0}$ such that $\widehat{M_{0}} \backslash M_{0}$ is a single point $\omega$, called the singularity of $M_{0}$.

This result will be proved in Section 2 using topological group theory. The crucial point of the proof is Lemma 2.7, which states that on a cone-like manifold, the distance from a fixed point to its image through any contracting homothety in $\Gamma$ is bounded (a fact which does not necessarily hold on the universal covering of $M_{0}$ ).

Theorem 1.4 can now be restated as follows: If the restricted Riemannian holonomy of a tame cone-like manifold is reducible, then the metric is flat.

The key point of the proof is to show the existence of families of complete integral leaves of any of the two integrable foliations (corresponding to the parallel splitting of the tangent bundle), all isometric to each other. On the other hand, we show that the homotheties of $\left(M_{0}, g_{0}\right)$ preserve these families, and we end up with pairs of complete Riemannian manifolds which are at the same time isometric and homothetic to each other, thus flat. 
Roughly, these ideas are inspired by the original proof of Gallot's theorem [6]. However, in our more general cone-like setting, the difficulty comes from the lack of information about the incomplete geodesics (which, for a cone, are just its rays, the orbits of the homothety flow). This is where we use the assumption that $D$ is a tame connection, which is equivalent to the existence of uniform bounds for the life-times of the incomplete geodesics generated by vectors belonging to any compact subset of the tangent bundle, and allows us to construct the families of complete submanifolds mentioned above.

Theorem 1.4 applies to a wide class of Weyl connections: We show in Section 5 that the tameness condition is fulfilled by any small deformations of a cone metric, and more generally by any Weyl connection $D$ which is stable with respect to a complete metric $g$ in the conformal class:

Theorem 1.6. A Weyl connection on a conformal manifold $(M, c)$ which is stable with respect to some complete metric $g \in c$ is tame.

The stability condition ( $c f$. Definition 5.1) is equivalent to a system of differential inequalities (Equations (12)-(13) below) and is therefore an open condition. It is satisfied by a $C^{1}$-neighborhood of the canonical Weyl connection on the quotient of a cone by one of its homotheties. Another class of stable Weyl connections is described in Example 5.7.

We classify, at last, all possible restricted holonomy groups of closed tame Weyl connections in Theorem 6.1. For some of these groups, the tameness condition turns out to be automatic. We give, moreover, a full geometrical description of the underlying manifolds in Theorem 6.2.

ACKnowledgment. We thank the anonymous referee for having pointed out an error in a previous version of this article and for several suggestions which significantly improved the exposition.

\section{The minimal Riemannian Cover of a Closed Weyl Connection}

In this section, $(M, c)$ denotes a connected conformal manifold and $D$ denotes a closed, nonexact, Weyl connection on $(M, c)$ (see e.g. [3] for the basic definitions). Let $\pi: \tilde{M} \rightarrow M$ be the universal cover of $M$, endowed with the induced conformal structure $\tilde{c}:=\pi^{*} c$, and Weyl derivative $\tilde{D}:=\pi^{*} D$. Since $\tilde{M}$ is simply connected, $\tilde{D}$ is exact, so $\tilde{M}$ carries a Riemannian metric $\tilde{g}_{0} \in \tilde{c}$, unique up to a multiplicative constant, whose Levi-Civita covariant derivative is just $\tilde{D}$.

Lemma 2.1. The group $\mathcal{A} \simeq \pi_{1}(M)$ of deck transformations of the covering $\tilde{M} \rightarrow M$ consists of homotheties of $\tilde{g}_{0}$.

Proof. Every element $\alpha \in \mathcal{A}$ is a conformal transformation of $(\tilde{M}, \tilde{c})$, so there exists a positive function $\rho$ such that $\alpha^{*} \tilde{g}_{0}=\rho^{2} \tilde{g}_{0}$. On the other hand, $\alpha$ preserves $\tilde{D}$, so the Riemannian metric $\alpha^{*} \tilde{g}_{0}$ is $\tilde{D}$-parallel, therefore $\rho$ is constant.

For every $\alpha \in \mathcal{A}$ we denote by $\rho(\alpha)$ the constant of homothety. Consider the sub-group of isometric deck transformations of $\left(\tilde{M}, \tilde{g}_{0}\right)$ :

$$
\mathcal{I}:=\{\alpha \in \mathcal{A} \mid \rho(\alpha)=1\} .
$$

Of course, $\rho$ being a group homomorphism from $(\mathcal{A}, \circ)$ to $\left(\mathbb{R}_{+}^{*}, \times\right), \mathcal{I}$ is a normal subgroup of $\mathcal{A}$. The quotient manifold $M_{0}:=\tilde{M} / \mathcal{I}$ is a Galois covering of $M$ with Abelian deck transformation group $\Gamma:=\mathcal{A} / \mathcal{I}$, isomorphic to the subgroup $\rho(\mathcal{A})$ of $\left(\mathbb{R}_{+}^{*}, \times\right)$. Moreover $\tilde{g}_{0}$ projects to a Riemannian 
metric $g_{0}$ on $M_{0}$. Clearly $\rho$ descends to a group homomorphism, also denoted by $\rho: \Gamma \rightarrow \mathbb{R}_{+}^{*}$, such that $f^{*} g_{0}=\rho(f)^{2} g_{0}$ for every $f \in \Gamma$. The pull-back of $D$ to $M_{0}$ (still denoted by $D$ ) is the Levi-Civita connection of $g_{0}$, and the deck transformation group $\Gamma$ acts by pure homotheties on $\left(M_{0}, g_{0}\right)$ (i.e. the only isometry in $\Gamma$ is the identity). This motivates the following:

Definition 2.2. Let $D$ be a closed Weyl connection on a connected conformal manifold $(M, c)$. The triple $\left(M_{0}, g_{0}, \Gamma\right)$, together with the covering $\pi: M_{0} \rightarrow M=M_{0} / \Gamma$ is called the minimal Riemannian cover of $(M, c, D)$.

Notice that there is no canonical way to choose $g_{0}$ in its homothety class, but all the properties we will consider in the sequel will not depend on such a choice.

If $d$ denotes the geodesic distance on $M_{0}$ induced by the Riemannian metric $g_{0}$, every $f \in \Gamma$ is a homothety of the metric space $\left(M_{0}, d\right)$, i.e. $d(f(x), f(y))=\rho(f) d(x, y)$ for each $x, y \in M_{0}$.

Definition 2.3. A cone-like space is a locally compact metric space $\left(M_{0}, d\right)$ together with a finitely generated, non-trivial group $\Gamma$ acting freely and properly discontinuously by homotheties on $\left(M_{0}, d\right)$, such that $\Gamma$ contains no isometry besides the identity, and such that the quotient $M_{0} / \Gamma$ is a compact topological space.

Remark 2.4. The above considerations show that the minimal Riemannian cover defines a oneto-one correspondence between the set of triples $(M, c, D)$ consisting in a compact manifold $M$, a conformal structure $c$ and a closed, non-exact Weyl connection $D$ on it, and the set of cone-like Riemannian manifolds $\left(M_{0}, g_{0}, \Gamma\right)$ (modulo constant rescalings of the metric $\left.g_{0}\right)$.

A fundamental example of cone-like space, which is the Leitfaden of our present study, is the following:

Example 2.5. Let $\left(N, g^{N}\right)$ be a complete Riemannian manifold and let

$$
\left(M_{0}, g_{0}\right):=\left(\mathbb{R}_{+}^{*} \times N, d t^{2}+t^{2} g^{N}\right)
$$

be the Riemannian cone over $N$ (note that $g_{0}$ and the product metric $g$ on $M_{0} \simeq \mathbb{R} \times N$ are conformally related by setting $t=e^{s}, t \in \mathbb{R}_{+}^{*}, s \in \mathbb{R}$ ). The multiplication by some $\lambda>1$ on the $\mathbb{R}$-factor is a strict homothety of $g_{0}$ and an isometry of $g$. It generates a group $\Gamma$ acting freely and properly discontinuously on $M_{0}$. The metric $g$ projects to the product metric, also denoted by $g$, on the quotient manifold $M:=M_{0} / \Gamma \simeq S^{1} \times N$. The Levi-Civita connection $D_{0}$ of $g_{0}$ is $\Gamma$-invariant, inducing therefore a closed, non-exact Weyl connection $D$ on $(M,[g])$. It is straightforward to check that $\left(M_{0}, g_{0}\right)$ is the minimal Riemannian cover of $(M,[g], D)$. The theorem of Gallot [6] about the irreducibility of the Riemannian cone $\left(\mathbb{R}_{+}^{*} \times N, d t^{2}+t^{2} g^{N}\right)$ is equivalent to the fact that $D$ is either flat or irreducible.

Metric cones can be equivalently characterized by the existence of a global homothetic gradient flow, i.e. a complete vector field which is locally a gradient (with respect to a local $D$-parallel metric $\left.g_{0}\right)$, and acts infinitesimally by homotheties of $g_{0}$. We will exhibit in this section some further properties which the class of cone-like spaces shares with the (much more restricted) class of Riemannian cones. The most important such property is that a cone-like space still has an "apex", more precisely, it can be completed by adding one point. This is exactly the statement of Theorem 1.5, which we will now prove. 
Proof of Theorem 1.5. Since every commutator of $\Gamma$ is an isometry of $\left(M_{0}, d\right)$, the hypothesis ensures that $\Gamma$ is Abelian. We need to show that $\left(M_{0}, d\right)$ contains at least one non-convergent Cauchy sequence, and that any two such sequences are equivalent.

Let $f \in \Gamma$ be any element with $\rho(f)<1$. For every $x \in M_{0}$ and $m<n \in \mathbb{N}$ we have

$$
d\left(f^{m}(x), f^{n}(x)\right) \leq \sum_{k=m}^{n-1} d\left(f^{k}(x), f^{k+1}(x)\right)=d(x, f(x)) \sum_{k=m}^{n-1} \rho(f)^{k}<d(x, f(x)) \frac{\rho(f)^{m}}{1-\rho(f)},
$$

thus showing that $\left\{f^{n}(x)\right\}$ is a Cauchy sequence. If this sequence had a limit $l$ in $M_{0}$, then $l$ would be a fixed point of $f$, contradicting the fact that $\Gamma$ acts freely. Thus $\left(M_{0}, d\right)$ is non-complete.

Lemma 2.6. Let $\left\{x_{n}\right\}$ be a non-convergent Cauchy sequence in $\left(M_{0}, d\right)$. Then there exists $x \in M_{0}$ and a sequence $\left\{f_{n}\right\}$ of elements of $\Gamma$ satisfying $\lim _{n \rightarrow \infty} \rho\left(f_{n}\right)=0$ such that $\left\{x_{n}\right\}$ is equivalent to $\left\{f_{n}(x)\right\}$.

Proof. Let $\pi$ denote the projection of $M_{0}$ onto the compact space $M:=M_{0} / \Gamma$. By choosing a subsequence if necessary, we may assume that $\pi\left(x_{n}\right)$ converges to some $y \in M$. Take $x \in \pi^{-1}(y)$. Since $\Gamma$ acts properly discontinuously, there exists some open neighborhood $U_{0}$ of $x$ such that $h\left(U_{0}\right) \cap U_{0}=\emptyset$ for every $h \in \Gamma$ different from the identity. We choose $r>0$ such that the ball $B_{x}(2 r)$ of radius $2 r$ in $x$ lies in $U_{0}$. Then $U:=\pi\left(B_{x}(r)\right)$ is a neighborhood of $y$ in the quotient topology, so there exists some $n_{0}$ such that $\pi\left(x_{n}\right) \in U$ for $n \geq n_{0}$. This shows that for $n \geq n_{0}$ there exist $z_{n} \in B_{x}(r)$ and $f_{n} \in \Gamma$ such that $x_{n}=f_{n}\left(z_{n}\right)$.

Suppose that $\rho\left(f_{n}\right)$ does not tend to zero. By taking a subsequence if necessary, we may assume that $\rho\left(f_{n}\right)>\delta$ for every $n$. For every $m, n$ such that $f_{n} \neq f_{m}$, the open balls $f_{n}\left(B_{x}(2 r)\right)$ and $f_{m}\left(B_{x}(2 r)\right)$ are disjoint, being included in $f_{n}\left(U_{0}\right)$, and $f_{m}\left(U_{0}\right)$ respectively. As $f_{n}\left(z_{n}\right) \in f_{n}\left(B_{x}(r)\right)$ and $f_{m}\left(z_{m}\right) \in f_{m}\left(B_{x}(r)\right)$, we get $d\left(x_{n}, x_{m}\right)=d\left(f_{n}\left(z_{n}\right), f_{m}\left(z_{m}\right)\right) \geq 2 r \delta$. The fact that $\left\{x_{n}\right\}$ is a Cauchy sequence ensures therefore the existence of an index $N$ such that $f_{n}=f_{N}$ for every $n>N$. Since $B_{x}(r)$ is relatively compact, we may assume (passing to some subsequence, if necessary) that $z_{n}$ tends to $z \in \overline{B_{x}(r)}$. Thus $\left\{x_{n}\right\}$ converges to $f_{N}(z)$, contradicting the fact that $\left\{x_{n}\right\}$ does not converge.

This shows that $\lim _{n \rightarrow \infty} \rho\left(f_{n}\right)=0$. Since $d\left(f_{n}\left(z_{n}\right), f_{n}(x)\right)=\rho\left(f_{n}\right) d\left(z_{n}, x\right)<r \rho\left(f_{n}\right)$, the sequences $\left\{x_{n}\right\}$ and $\left\{f_{n}(x)\right\}$ are equivalent, thus proving the lemma.

In order to conclude the proof of the theorem we need one more technical result.

Lemma 2.7. For every fixed point $x \in M_{0}$ there exists a constant $K_{x}$, depending on $x$, such that $d(x, f(x))<K_{x}$ for every contracting $f \in \Gamma$ (i.e. with $\rho(f)<1$ ).

Proof. Let $\left\{h_{1}, \ldots, h_{n}\right\}$ be a system of generators of $\Gamma$ with $\rho_{i}:=\rho\left(h_{i}\right)>1$ and let

$$
D_{x}:=\max _{\{i=1, \ldots, n\}} d\left(x, h_{i}(x)\right) .
$$

For every $\left(a_{1}, \ldots, a_{k}\right) \in \mathbb{N}^{k}$, we claim that

$$
d\left(x,\left(\prod_{i=1}^{k} h_{i}^{a_{i}}\right)(x)\right) \leq D_{x} \prod_{i=1}^{k} \frac{\rho_{i}^{a_{i}+1}-1}{\rho_{i}-1} .
$$


We prove the claim by induction on $k$. For $k=1$ we have

$$
d\left(x, h_{1}^{a_{1}}(x)\right) \leq \sum_{s=0}^{a_{1}-1} d\left(h_{1}^{s}(x), h_{1}^{s+1}(x)\right)=d\left(x, h_{1}(x)\right) \sum_{s=0}^{a_{1}-1} \rho_{1}^{s} \leq D_{x} \frac{\rho_{1}^{a_{1}}-1}{\rho_{1}-1}<D_{x} \frac{\rho_{1}^{a_{1}+1}-1}{\rho_{1}-1} .
$$

Assume now that (1) holds for each $k \leq l$ and for every $\left(a_{1}, \ldots, a_{k}\right) \in \mathbb{N}^{k}$ and consider some element $\left(a_{1}, \ldots, a_{l+1}\right) \in \mathbb{N}^{l+1}$. We denote by

$$
h:=\prod_{i=1}^{l} h_{i}^{a_{i}} \text { and by } y_{j}:=\left(h_{l+1}^{j} \circ h\right)(x), \forall j=0, \ldots, a_{l+1} .
$$

Using (1) for $k=l$ we have

$$
d\left(x, y_{0}\right) \leq D_{x} \prod_{i=1}^{k} \frac{\rho_{i}^{a_{i}+1}-1}{\rho_{i}-1}
$$

and $d\left(y_{j}, y_{j+1}\right)=\rho_{l+1}^{j} d\left(y_{0}, y_{1}\right)=\rho(h) \rho_{l+1}^{j} d\left(x, h_{l+1}(x)\right)$, which further imply

$$
\begin{aligned}
d\left(x, \prod_{i=1}^{l+1} h_{i}^{a_{i}}(x)\right) & =d\left(x, y_{a_{l+1}}\right) \leq d\left(x, y_{0}\right)+\sum_{j=0}^{a_{l+1}-1} d\left(y_{j}, y_{j+1}\right) \\
& \leq D_{x} \prod_{i=1}^{l} \frac{\rho_{i}^{a_{i}+1}-1}{\rho_{i}-1}+D_{x} \prod_{i=1}^{l} \rho_{i}^{a_{i}} \sum_{j=0}^{a_{l+1}-1} \rho_{l+1}^{j} \\
& \leq D_{x} \prod_{i=1}^{l} \frac{\rho_{i}^{a_{i}+1}-1}{\rho_{i}-1}\left(1+\sum_{j=0}^{a_{l+1}-1} \rho_{l+1}^{j}\right) \leq D_{x} \prod_{i=1}^{l} \frac{\rho_{i}^{a_{i}+1}-1}{\rho_{i}-1}\left(\sum_{j=0}^{a_{l+1}} \rho_{l+1}^{j}\right) \\
& =D_{x} \prod_{i=1}^{l+1} \frac{\rho_{i}^{a_{i}+1}-1}{\rho_{i}-1}
\end{aligned}
$$

thus proving our claim for $k=l+1$. In order to finish the proof of the lemma, let $f \in \Gamma$ be an element with $\rho(f)<1$. By reordering the system of generators if necessary, we can write

$$
f=\prod_{i=1}^{n} h_{i}^{a_{i}}, \quad \text { with } a_{i} \geq 0 \text { for } i \leq m \text { and } a_{i} \leq 0 \text { for } i \geq m+1 .
$$

We denote $b_{i}:=-a_{i} \geq 0$ for $i \geq m+1$. Using (1) we obtain

$$
\begin{aligned}
d(x, f(x)) & =\left(\prod_{i=m+1}^{n} \rho_{i}^{a_{i}}\right) d\left(\prod_{i=1}^{m} h_{i}^{a_{i}}(x), \prod_{i=m+1}^{n} h_{i}^{b_{i}}(x)\right) \\
& \leq\left(\prod_{i=m+1}^{n} \rho_{i}^{a_{i}}\right)\left(d\left(x, \prod_{i=1}^{m} h_{i}^{a_{i}}(x)\right)+d\left(x, \prod_{i=m+1}^{n} h_{i}^{b_{i}}(x)\right)\right) \\
& \leq\left(\prod_{i=m+1}^{n} \rho_{i}^{a_{i}}\right)\left(D_{x} \prod_{i=1}^{m} \frac{\rho_{i}^{a_{i}+1}-1}{\rho_{i}-1}+D_{x} \prod_{i=m+1}^{n} \frac{\rho_{i}^{b_{i}+1}-1}{\rho_{i}-1}\right)
\end{aligned}
$$


We neglect the -1 terms in the numerators above and multiply the brackets. Remembering that $\prod_{i=1}^{n} \rho_{i}^{a_{i}}=\rho(f)<1$, we finally get

$$
\begin{aligned}
d(x, f(x)) & \leq D_{x}\left(\prod_{i=1}^{m} \frac{\rho_{i}}{\rho_{i}-1} \prod_{i=1}^{n} \rho_{i}^{a_{i}}+\prod_{i=m+1}^{n} \frac{\rho_{i}}{\rho_{i}-1}\right) \\
& \leq D_{x}\left(\prod_{i=1}^{m} \frac{\rho_{i}}{\rho_{i}-1}+\prod_{i=m+1}^{n} \frac{\rho_{i}}{\rho_{i}-1}\right) \\
& \leq D_{x}\left(\prod_{i=1}^{n} \frac{\rho_{i}}{\rho_{i}-1}+1\right)=: K_{x}
\end{aligned}
$$

where the last inequality follows from the fact that $a+b \leq a b+1$ for all $a, b \geq 1$.

Let now $\left\{x_{n}\right\}$ be a non-convergent Cauchy sequence in $M_{0}$. Choose $y \in M_{0}$ and $f \in \Gamma$ such that $\rho:=\rho(f)<1$. We claim that $\left\{x_{n}\right\}$ is equivalent to $\left\{f^{n}(y)\right\}$. By Lemma 2.6, there exists $x \in M_{0}$ and a sequence $\left\{f_{n}\right\}$ of elements of $\Gamma$ satisfying $\lim _{n \rightarrow \infty} \rho\left(f_{n}\right)=0$, such that $\left\{x_{n}\right\}$ is equivalent to $\left\{f_{n}(x)\right\}$. Since $\lim _{n \rightarrow \infty} \rho\left(f_{n}\right)=0$, there exists an increasing sequence of integers $\left\{k_{n}\right\}$ such that $\rho\left(f_{k_{n}}\right)<\rho^{n}$. As $\rho\left(f^{-n} \circ f_{k_{n}}\right)<1$, Lemma 2.7 yields

$$
d\left(f^{n}(x), f_{k_{n}}(x)\right)=\rho^{n} d\left(x,\left(f^{-n} \circ f_{k_{n}}\right)(x)\right) \leq K_{x} \rho^{n} .
$$

The sequences $\left\{f_{k_{n}}(x)\right\}$ and $\left\{f^{n}(x)\right\}$ are thus equivalent, so the same holds for $\left\{x_{n}\right\}$ and $\left\{f^{n}(x)\right\}$. Finally, for any $y \neq x,\left\{f^{n}(x)\right\}$ is clearly equivalent to $\left\{f^{n}(y)\right\}$, thus finishing the proof of the theorem.

Theorem 1.5 shows that, despite the fact that cone-like spaces only carry a discrete group of homotheties, they still have the one-point completion like all Riemannian cones. Note that the universal covering of a Riemannian cone is a Riemannian cone itself, therefore admits the onepoint completion as well. It is unknown whether this fact holds for the universal covering of an arbitrary cone-like space (see Section 6.4).

Functions on a Riemannian cone measuring geometric quantities like lengths, are equivariant with respect to the radial flow (acting by homotheties), and thus vary linearly on the rays.

In the more general case of cone-like spaces, we introduce, for further use, the following simple notion:

Definition 2.8. Two positive functions $f_{1}, f_{2}: M_{0} \rightarrow \mathbb{R}_{+}^{*}$ are said to be equivalent if their ratio is bounded above and below by positive constants. A function which is equivalent to the distance to the singularity $\omega$ is called quasi-linear.

Denote by $\delta: M_{0} \rightarrow \mathbb{R}_{+}^{*}$ the distance to the singularity $\omega \in \widehat{M}_{0}: \delta(x):=d(x, \omega)$.

Lemma 2.9. Let $\psi: M_{0} \rightarrow \mathbb{R}_{+}^{*}$ be any $\Gamma$-equivariant function of weight 1 on $M_{0}$ (i.e. satisfying $\psi \circ f=\rho(f) \psi$ for every element $f \in \Gamma$ ), such that $\psi$ and $\frac{1}{\psi}$ are locally bounded (e.g., $\psi$ is continuous). Then $\psi$ is quasi-linear. 
Proof. Consider a compact fundamental domain $\Omega$ of the action of $\Gamma$ on $M_{0}$ and define

$$
k_{1}:=\inf _{x \in \Omega} \frac{\psi(x)}{\delta(x)}, \quad k_{2}:=\sup _{x \in \Omega} \frac{\psi(x)}{\delta(x)} .
$$

Because $\delta$ is continuous and $\psi$ and its inverse are locally bounded, their quotients $\delta / \psi$ and $\psi / \delta$ are bounded on the compact set $\Omega$. It follows that $k_{1}, k_{2}$ are positive real numbers, so that

$$
\frac{\psi(x)}{\delta(x)} \in\left[k_{1}, k_{2}\right]
$$

holds tautologically on $\Omega$. Let now $y$ be an arbitrary point of $M_{0}$ and $f \in \Gamma$ such that $x:=f^{-1}(y) \in$ $\Omega$. From the equivariance property of $\psi$ we get

$$
\frac{\psi(y)}{\delta(y)}=\frac{\psi(f(x))}{\delta(f(x))}=\frac{\rho(f) \psi(x)}{\rho(f) \delta(x)}=\frac{\psi(x)}{\delta(x)} \in\left[k_{1}, k_{2}\right],
$$

which finishes the proof.

As a consequence of the previous lemma, we show for later use that if $\left(M_{0}, g_{0}\right)$ is the minimal Riemannian cover of a closed non-exact Weyl connection $D$ on a compact conformal manifold $(M, c)$, then any conformal factor relating $g_{0}$ to the pull-back on $M_{0}$ of a metric in the conformal class $c$ on $M$ is equivalent to the distance function $\delta$ to the singularity $\omega \in M_{0}$ :

Lemma 2.10. Let $g$ be the pull-back to $M_{0}$ of a metric in $c$ on $M$ and let $\varphi: M_{0} \rightarrow \mathbb{R}_{+}^{*}$ be defined by $g_{0}=\varphi^{2} g$. The function $\varphi$ is then quasi-linear on $M_{0}$.

Proof. Every element $f \in \Gamma$ being an isometry of $g$, we obtain

$$
\rho(f)^{2} \varphi^{2} g=\rho(f)^{2} g_{0}=f^{*} g_{0}=(\varphi \circ f)^{2} g,
$$

showing that $\varphi \circ f=\rho(f) \varphi$. The assertion thus follows from Lemma 2.9.

\section{TAME CONNECTIONS AND THEIR GEODESICS}

In contrast to the Riemannian situation, a Weyl connection on a compact conformal manifold is not necessarily geodesically complete.

Example 3.1. Let $(M, c)$ be a compact conformal manifold and let $D$ be a closed, non-exact, Weyl connection on $M$. Theorem 1.5 shows that the minimal Riemannian cover $\left(M_{0}, g\right)$ of $(M, c, D)$ is incomplete, so through every point of $M_{0}$ passes an incomplete geodesic. Its projection onto $M$ is thus an incomplete geodesic of $D$.

In order to study the geometry of $M_{0}$ in the neighborhood of its singularity $\omega$, we need to understand the behavior of the geodesics passing through or near $\omega$. In principle, the dynamics of the geodesic flow of $(M, g)$ can be rather wild near $\omega$. Here is a list of phenomena which may occur:

(1) The lengths of the geodesics starting at some given point $P$ and passing through $\omega$ (i.e. the life-time of an incomplete geodesic) might not be bounded.

(2) There might exist closed geodesics through $\omega$ (i.e. geodesics having finite life-time in both directions).

(3) There might even exist a complete geodesic whose adherence contains $\omega$. 
3.1. Tame connections. To begin with, let us recall some basic facts about the geodesic flow of an affine connection $D$ on a manifold $M$, or, equivalently, the exponential map

$$
\exp ^{D}: \mathcal{U} \rightarrow M \text {. }
$$

defined on a (maximal) open subset $\mathcal{U}$ of $T M$. For $X \in \mathcal{U}_{x}:=\mathcal{U} \cap T_{x} M$, $\exp ^{D}(X)$ is the point $\gamma(1)$ on the geodesic defined by

$$
\gamma(0)=x \text { and } \dot{\gamma}(0)=X
$$

We define the life-time $\mathcal{L}^{D}: T M \rightarrow(0,+\infty]$ of a half-geodesic generated by $X \in T M$, by

$$
\mathcal{L}^{D}(X):=\sup \{t>0 \mid t X \in \mathcal{U}\},
$$

in other words, the supremum of the time for which the half-geodesic tangent to $X$ is defined. Of course, if $(M, D)$ is geodesically complete, all life-times are infinite.

We split the complement $T M \backslash\{0\}$ of the zero section in the tangent bundle into two sets, the set $\mathcal{I}^{D}$ of vectors generating incomplete half-geodesics, and its complement $\mathcal{C}^{D}$. These subsets are both star-shaped, i.e. for a vector $X \in T M$

$$
X \in \mathcal{I}^{D} \Longleftrightarrow s X \in \mathcal{I}^{D}, \quad \forall s>0 .
$$

The two sets $\mathcal{I}^{D}$ and $\mathcal{C}^{D}$ are in general neither open, nor closed. They are however Borel measurable, as $\mathcal{C}^{D}$ is an infinite intersection of open sets.

For the following definition, we need to consider a Riemannian metric $g$ on $M$, in order to define the sphere bundle $S^{g} M$ of unit vectors on $M$. However, the notion that we are about to define does not depend on the choice of such a metric:

Definition 3.2. A connection $D$ on $M$ is tame if and only if the function

$$
\mu^{g}: M_{0} \rightarrow[0,+\infty], \quad \mu^{g}(x):=\sup \left\{\{0\} \cup\left\{\mathcal{L}^{D}(X) \mid X \in \mathcal{I}_{x}^{D} \cap S_{x}^{g} M\right\}\right\}
$$

is locally bounded on $M$.

Of course, $\mu^{g}=0$ if and only if $D$ is geodesically complete.

If $D:=\nabla^{g}$ is the Levi-Civita connection of a Riemannian manifold $(M, g), \mu^{g}(x)$ is the supremum of the lengths of all incomplete half-geodesics starting in $x$. If $\nabla^{g}$ is tame, we say that $(M, g)$ is a tame Riemannian manifold. Note that, while the tameness of the connection $D$ does not depend on the auxiliary metric in Definition 3.2, the tameness of a Levi-Civita connection depends on the corresponding metric (see Example 6.5 for an example of a non-tame Riemannian manifold, which becomes complete after a conformal rescaling).

3.2. Tame cone-like spaces. If $D$ is a connection on $M$, the induced connection (still denoted by $D$ ) on some covering $M_{0}$ of $M$ is tame if and only if $D$ is tame on $M$. A tame closed Weyl connection $D$ on a compact manifold $(M, c)$ is therefore equivalent to a tame cone-like Riemannian manifold $\left(M_{0}, g_{0}\right)$. Here we have the following criterion:

Proposition 3.3. A cone-like manifold $\left(M_{0}, g_{0}\right)$ is tame if and only if $\mu: M_{0} \rightarrow(0,+\infty]$ is (finite and) quasi-linear on $M_{0}$, i.e. if there exists a constant $K>0$ such that

$$
\delta(x) \leq \mu(x) \leq K \delta(x), \forall x \in M_{0},
$$

where $\delta: M_{0} \rightarrow \mathbb{R}_{+}^{*}$ is the distance from a point to the singularity $\omega$. 
Proof. The distance $\delta$ to the singularity $\omega \in M_{0}$ is always continuous on $M_{0}$, and $\mu \geq \delta$, therefore $\frac{1}{\mu}$ is locally bounded. On the other hand, $\delta$ and $\mu$ are clearly $\Gamma$-equivariant of weight 1 , because both denote geometrical lengths. Therefore, if (2) holds on a fundamental domain of $\Gamma$, then it holds on whole $M_{0}$. As such a fundamental domain is relatively compact, the quasi-linearity of $\mu$ is thus equivalent to its local boundedness.

It is thus obvious that a cone over a complete Riemannian manifold is tame (its only incomplete geodesics are its rays, thus $\mu=\delta$ ). On the other hand, not all cone-like manifolds are tame, as we will see in Section 6.2, therefore not all closed Weyl connections on a compact manifold are tame. We will however show in that the tameness condition holds for an open set of Weyl connections (in the $C^{1}$-topology) containing the ones constructed (as in Example 2.5) from Riemannian cones.

\section{Closed Weyl connections With Reducible holonomy}

The goal of this section is to prove our main result, Theorem 1.4, concerning the holonomy of tame locally metric connections. We will rephrase it in terms of Weyl connections.

Theorem 4.1. If the restricted holonomy representation of a closed, non-exact, tame Weyl connection $D$ on a compact conformal manifold $(M, c)$ is reducible, then $D$ is flat.

Proof. We start by showing that if the restricted holonomy $\operatorname{Hol}_{0}(D)$ is reducible, then there exists a finite covering $\bar{M}$ of $M$ on which the full holonomy of the pull-back of $D$ has reducible holonomy. In order to keep the argument as simple as possible, we will not be very precise on the holonomy groups and consider them as abstract groups rather than as transformation groups of each tangent space.

Consider the metric $\tilde{g}_{0}$ on the universal cover $\tilde{M}$ of $M$ (defined up to a multiplicative constant), whose Levi-Civita covariant derivative $\tilde{\nabla}$ is the pull-back of $D$ to $\tilde{M}$. The holonomy of $\tilde{\nabla}$ is clearly equal to the restricted holonomy of $D$. By Theorem IV.5.4 in [10], the tangent bundle of $\tilde{M}$ splits in a direct sum $T \tilde{M}=T_{0} \oplus \ldots \oplus T_{m}$ of $\tilde{\nabla}$-parallel sub-bundles and the holonomy group of $\tilde{\nabla}$ satisfies $\operatorname{Hol}(\tilde{\nabla})=H_{1} \times \ldots \times H_{m}$, where $H_{i}$ acts irreducibly on $T_{i}$ and trivially on $T_{j}$ for $j \neq i\left(T_{0}\right.$ being the flat component). Moreover this decomposition is unique up to a permutation of the set $\{1, \ldots, m\}$ (such permutations may occur if some of the factors $H_{i}$ coincide).

By Lemma 2.1, every element $f \in \mathcal{A}$ of the deck transformation group of the covering $\tilde{M} \rightarrow M$ is affine with respect to $\tilde{\nabla}$, so there exists a permutation $\sigma_{f}$ of $\{1, \ldots, m\}$ such that $f_{*}\left(T_{i}\right)=T_{\sigma_{f}(i)}$. Let $\mathcal{B} \subset \mathcal{A}$ be the kernel of the group homomorphism $\mathcal{A} \rightarrow S_{m}$ given by $f \mapsto \sigma_{f}$. The metric $\tilde{g}_{0}$ and the connection $\tilde{\nabla}$ on $\tilde{M}$ descend to a conformal structure $\bar{c}$ and a Weyl connection $\bar{D}$ on the quotient $\bar{M}:=M / \mathcal{B}$, which is a finite covering of $M$ with group $\mathcal{A} / \mathcal{B} \subset S_{m}$. By construction, the holonomy group of $\bar{D}$ on $\bar{M}$ is reducible.

Replacing $(M, c, D)$ by $(\bar{M}, \bar{c}, \bar{D})$, we can from now on assume that the full holonomy of $D$ is reducible. This implies that the tangent bundle of the minimal Riemannian cover $\left(M_{0}, g_{0}\right)$ of $(M, c, D)$ splits in a direct sum of orthogonal distributions $T M=V_{1} \oplus V_{2}$, parallel with respect to the Levi-Civita connection $\nabla^{0}=D$ of $g_{0}$. These distributions are integrable, hence define two orthogonal (and complementary) foliations on $M_{0}$.

We will use the notion maximal leaf $M_{i}, i=1,2$, through $x \in M_{0}$ to denote the set of points that can be connected to $x$ by means of a smooth curve tangent to $V_{i}$. It is a standard fact that 
$M_{i}$ have smooth structures such that $M_{i} \rightarrow M_{0}$ are immersions (although $M_{i}$ are not necessarily submanifolds of $M_{0}$ ).

We start with two preliminary results which hold on every (not necessarily complete) reducible Riemannian manifold $\left(M_{0}, g_{0}\right)$. The first one is an elementary consequence of the local de Rham decomposition theorem.

Lemma 4.2. Let $U_{1}$ be a local leaf of $V_{1}$ (connected but not necessarily complete) and assume that $X \in \Gamma\left(V_{2}\right)$ is a parallel vector field along $U_{1}$. Assume moreover that $\exp _{x} t X$ is defined for all $x \in U_{1}$ and $t \in[0,1]$. Then $x \mapsto \psi(x):=\exp _{x} X$ maps $U_{1}$ locally isometrically onto its image $U_{1}^{\prime}$ (which is itself a leaf of $V_{1}$ ).

Proof. Consider the map $\varphi: U_{1} \times[0,1] \rightarrow M_{0}$ defined by $\varphi(x, t):=\exp _{x} t X$. Define $X_{(x, t)} \in$ $T_{\varphi(x, t)} M_{0}$ by

$$
X_{(x, t)}:=\left.\frac{d}{d s}\right|_{s=t} \varphi(x, s) .
$$

In other words, $X_{(x, t)}$ is the tangent vector to the geodesic $s \rightarrow \exp _{x} s X$ at $s=t$, so we clearly have the relation

$$
\varphi(x, t+s)=\exp _{\varphi(x, t)} s X_{(x, t)} .
$$

Let us fix $x \in U_{1}$ and denote $x_{t}:=\varphi(x, t)$. The local de Rham decomposition theorem (Proposition IV.5.2 in [10]) states that each $x_{t}$ has a neighborhood $U(t)$ in $M_{0}$ isometric to a Riemannian product $U(t) \simeq U_{1}(t) \times U_{2}(t)$, where $U_{1}(t)$ and $U_{2}(t)$ are local leaves of $V_{1}$ and $V_{2}$ through $x_{t}$.

The geodesic segment $\varphi(\{x\} \times[0,1])$ is compact, so it can be covered by a finite number of neighborhoods $U_{2}\left(s_{1}\right), \ldots, U_{2}\left(s_{n}\right)$, with $0=s_{1}<\ldots<s_{n}=1$. Choose now $t_{i} \in\left(s_{i}, s_{i+1}\right) \forall i=$ $1, \ldots, n-1$, such that $\varphi\left(x, t_{i}\right) \in U_{2}\left(s_{i}\right) \cap U_{2}\left(s_{i+1}\right)$, and set $t_{n}:=1$. For $k=1, \ldots, n$, let $V_{k}$ be the open subset of $U_{1}$ defined by

$$
V_{k}:=\left\{y \in U_{1} \mid \varphi\left(y, s_{k}\right) \in U\left(s_{k}\right)\right\} .
$$

We denote by $V$ the intersection of the $V_{k}$ 's and by $W_{k}$ the subset of $U\left(t_{k}\right)$ given by $W_{k}:=$ $\varphi\left(V \times\left\{t_{k}\right\}\right)$.

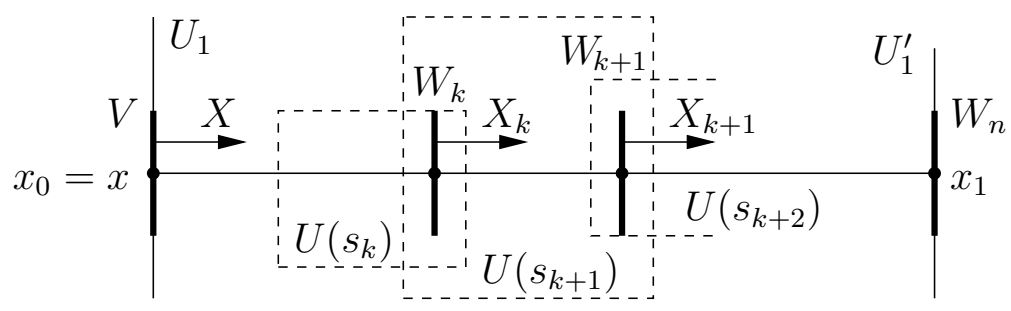

Figure 1. Stepwise exponentiation along the geodesic segment $\varphi(\{x\} \times[0,1])$.

Consider the vector field $X_{k}$ along $W_{k}$ whose value at $\varphi\left(y, t_{k}\right)$ is $X_{\left(y, t_{k}\right)}$. By construction, there exists a bijection $\varphi_{k}: W_{k} \rightarrow W_{k+1}$ defined by $\varphi_{k}\left(\varphi\left(y, t_{k}\right)\right):=\varphi\left(y, t_{k+1}\right)$ for all $y \in V$.

We claim that for every $k=1, \ldots, n$,

(1) $\varphi_{k-1}$ is an isometry;

(2) the vector field $X_{k}$ is parallel along $W_{k}$; 
(3) $W_{k}$ is an open subset of the leaf $U_{1}\left(t_{k}\right)$.

For $k=1$, the first statement is empty, and the other two hold by hypothesis.

Assume that the claim holds for some $k \geq 1$. Since $X_{k}$ is parallel along $W_{k}$, it is constant in the product coordinates on $W_{k} \times U_{2}\left(s_{k}\right) \subset U\left(s_{k}\right)$ (i.e. there exists $Z \in T_{x_{t_{k}}} U_{2}\left(s_{k}\right)$ such that $\left.\left(X_{k}\right)_{\left(z, x_{t_{k}}\right)}=(0, Z), \forall z \in W_{k}\right)$. By (3) we have $\varphi_{k}\left(\varphi\left(y, t_{k}\right)\right)=\exp _{\varphi\left(y, t_{k}\right)}\left(t_{k+1}-t_{k}\right) X_{\left(y, t_{k}\right)}$ so in the product coordinates $\varphi_{k}\left(z, x_{t_{k}}\right)=\left(z, x_{t_{k+1}}\right)$ for all $z \in W_{k}$, showing that $W_{k} \subset U_{1}\left(t_{k}\right)$ and that $\varphi_{k}$ is an isometry. Moreover $X_{k+1}$ is constant along $W_{k+1}$ in these coordinates, thus proving the induction step.

We have shown that in the neighborhood $V$ of $x$ in $U_{1}$, the map $x \mapsto \psi(x)=\exp _{x} X$ is the composition of $n-1$ isometries $\varphi_{n-1} \circ \cdots \circ \varphi_{1}$ between local leaves of the distribution $V_{1}$. As this holds in the neighborhood of every point $x$ of $U_{1}, \psi$ is a local isometry from $U_{1}$ to its image $U_{1}^{\prime}$. In particular, this shows that $U_{1}^{\prime}$ is an open subset of the complete integral leaf of $V_{1}$ passing through $\varphi(x, 1)$.

The next result, which is elementary as well, shows that exponentiating a geodesic tangent to $V_{1}$ in the direction of a constant or affine Jacobi field tangent to $V_{2}$ yields another geodesic whenever it is defined.

Lemma 4.3. Let $\gamma:[a, b] \rightarrow M_{0}$ be a geodesic tangent to $V_{1}$ parametrized by arc-length and let $X \in T_{\gamma(a)} M_{0}$ be a vector tangent to $V_{2}$. Extend $X$ to a parallel vector field along $\gamma$.

(i) Assume that $\gamma_{s}(t):=\exp _{\gamma(t)}(s X)$ is well-defined for all $t \in[a, b]$ and $s \in[0,1]$. Then $\gamma_{1}(t)$ is a geodesic in $M_{0}$ and its tangent vector at $t$ is the parallel transport of $\dot{\gamma}(t)$ at $\exp _{\gamma(t)}(X)$ along the geodesic $s \mapsto \exp _{\gamma(t)}(s X)$.

(ii) Assume that $\gamma^{X}(t):=\exp _{\gamma(t)}(t X)$ is well-defined for all $t \in[a, b]$. Then $\gamma^{X}(t)$ is a geodesic in $M_{0}$ and the projections of $\dot{\gamma}^{X}(t)$ onto $V_{1}$ and $V_{2}$ are parallel vector fields along $\gamma^{X}$ of length 1 and $|X|$ respectively.

Proof. (i) The first statement follows immediately from Lemma 4.2. The second one is a consequence of the inductive claim used to prove the same lemma.

(ii) Assume first that $M_{0}$ is a global Riemannian product $M_{0}=M_{1} \times M_{2}$. If $\gamma(a)=\left(m_{1}, m_{2}\right)$, then $\gamma(t)=\left(\gamma_{1}(t), m_{2}\right)$ for some geodesic $\gamma_{1}$ in $M_{1}$ parametrized by arc-length. The vector field $X$ along $\gamma$ can be written $X=\left(0, X_{2}\right)$, where $X_{2}$ is a constant vector tangent to $M_{2}$ at $m_{2}$. Denoting by $\gamma_{2}(t)=\exp _{m_{2}} t X_{2}$ the geodesic in $M_{2}$ starting at $m_{2}$ with initial speed $X_{2}$, then $\gamma^{X}(t)=\left(\gamma_{1}(t), \gamma_{2}(t)\right)$, is a geodesic in $M_{0}$. The projections of $\dot{\gamma}^{X}(t)$ onto $V_{1}$ and $V_{2}$ are $\left(\dot{\gamma}_{1}, 0\right)$ and $\left(0, \dot{\gamma}_{2}\right)$, which are clearly parallel vector fields along $\gamma^{X}$ of length 1 and $|X|$ respectively.

Back to the general case, it is of course enough to show that the statement holds in the neighborhood of every point $\gamma^{X}\left(t_{0}\right)$. Since the domain of definition of the exponential on the normal bundle of a geodesic is open, the curve $c(t):=\exp _{\gamma(t)}\left(t_{0} X\right)$ is well-defined for $t$ near $t_{0}$. By Lemma 4.2, $c(t)$ is a geodesic through $x:=\gamma^{X}\left(t_{0}\right)$, parametrized by arc-length. Moreover, if $Y$ denotes the parallel vector field along $c(t)$ with $Y_{x}=d \exp _{\gamma\left(t_{0}\right)}\left(t_{0} X\right)$, Lemma 4.2 also shows that $Y_{\gamma(t)}=d \exp _{\gamma(t)}\left(t_{0} X\right)$, so by $(3), \gamma^{X}(t)=\exp _{\gamma(t)}\left(\left(t-t_{0}\right) Y\right)$. 


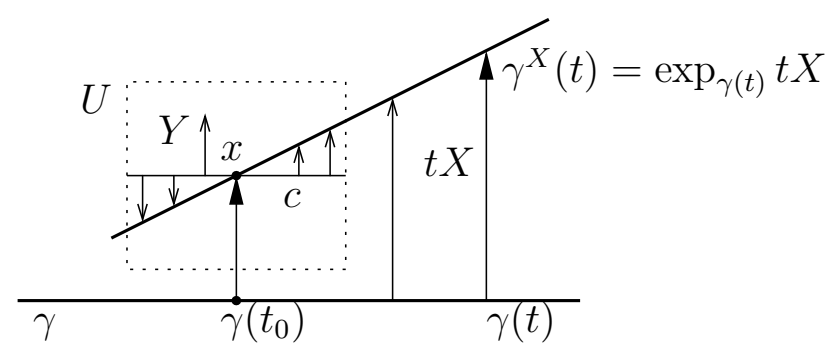

Figure 2. Idea of the proof of Lemma 4.3 (ii).

By the local de Rham theorem, the point $x$ has a neighborhood $U$ isometric to $U_{1} \times U_{2}$, where $U_{i}$ is some local leaf of $V_{i}$ through $x$. As $\gamma^{X}(t)$ lies in $U$ for $t$ near $t_{0}$, the statement follows from the first part of the proof.

We assume from now on that $D$ is a closed tame Weyl connection on a compact conformal manifold $(M, c)$ which has reducible holonomy and that $\left(M_{0}, g_{0}\right)$ is the minimal Riemannian cover of $(M, c, D)$. We denote as before by $d$ the distance induced by $g_{0}$ on $M_{0}$, by $\omega$ the singularity of $M_{0}$ and by $\delta$ the distance to the singularity: $\delta(x):=d(x, \omega)$. Since $D=\nabla^{0}, g_{0}$ has reducible holonomy, so the above results apply to the present setting. We will need the following quantitative version of the local de Rham decomposition theorem for $\left(M_{0}, g_{0}\right)$.

Lemma 4.4. There exists a quasi-linear function $\sigma: M_{0} \rightarrow \mathbb{R}_{+}^{*}$ such that each point $x \in M_{0}$ has a neighborhood $U$ and an isometry $F: U \rightarrow B_{x}^{1}(\sigma(x)) \times B_{x}^{2}(\sigma(x))$, with $F(x)=(x, x)$, where $B_{x}^{i}(r)$ is the ball of radius $r$ around $x$ in the maximal leaf $M_{i}$ though $x$, tangent to the distribution $V_{i}$.

Proof. By Lemma 2.9, it is enough to define $\sigma$ on a relatively compact fundamental domain $K \subset M_{0}$ of the covering $M_{0} \rightarrow M$, and to extend it to $M_{0}$ in a $\Gamma$-equivariant way by $\sigma(f(x))=\rho(f) \sigma(x)$ for all $f \in \Gamma$ and $x \in K$.

The local de Rham theorem ensures that for every $x \in M_{0}$ there exist neighborhoods $U_{i}(x)$ of $x$ in the maximal leaf $M_{i}(x)$ though $x$, tangent to the distribution $V_{i}$, such that $U_{1}(x) \times U_{2}(x)$ is isometric to a neighborhood $U(x)$ of $x$ in $M_{0}$. Take a finite number of points $x_{i}$ such that $\bar{K} \subset \cup_{i} U\left(x_{i}\right)$. Each neighborhood $U_{1}\left(x_{i}\right)$ and $U_{2}\left(x_{i}\right)$ contains a geodesic ball centered in $x_{i}$ of radius $r_{1}\left(x_{i}\right)$ and $r_{2}\left(x_{i}\right)$ respectively. It is then enough to define $\sigma$ on $K$ to be the minimum of all these radii.

We now come to a key point of the proof of Theorem 4.1, namely the existence of complete maximal leaves tangent to the distributions $V_{i}$.

Proposition 4.5. If $M_{1}$ is a maximal leaf of $V_{1}$ which is incomplete, then every maximal leaf of $V_{2}$ which intersects $M_{1}$ is complete.

Proof. Since $M_{1}$ is totally geodesic and incomplete, through every point $x \in M_{1}$ passes a geodesic $\gamma:(0, r] \rightarrow M_{1}$ parametrized by arc-length, such that $\gamma(r)=x$, which can not be defined at $t=0$. Since $M_{i}$ is totally geodesic in $M_{0}, \gamma$ is also a geodesic in $M_{0}$. By Theorem 1.5, we must have $\lim _{t \rightarrow 0} \gamma(t)=\omega$ in $\left(\widehat{M}_{0}, d\right)$.

Let $X \in T_{x} M \cap V_{2}$ be any unit normal vector to $M_{1}$ at $x$, extended as before to a parallel vector field along $\gamma$. We claim that the geodesic generated by $X$ in $M_{0}$ is complete. 
The crucial point here is the fact that every point $\gamma(t)$ is far enough from the singularity $\omega$, in order to ensure that the exponential function is well-defined in a suitable neighborhood. More precisely, Proposition 3.3 shows that there exists a constant $\kappa>0$ such that for every $t \in(0, r]$, the distance $\delta(\gamma(t))$ from $\gamma(t)$ to $\omega$ (in $M_{0}$ ) is bounded from below by $\kappa t$. Consequently, $\exp _{\gamma(t)} s X$ is well-defined for $|s| \leq \kappa t$, so by Lemma 4.3 (ii), the curve $\gamma_{1}:(0, r] \rightarrow M_{0}$ defined by $\gamma_{1}(t):=$ $\exp _{\gamma(t)} \kappa t X$ is a geodesic in $M_{0}$ with $\left|\dot{\gamma}_{1}\right|^{2}=1+\kappa^{2}$. Moreover, the limit in $\widehat{M_{0}}$ of $\gamma_{1}(t)$ as $t \rightarrow 0$ is clearly $\omega$. Proposition 3.3 applied this time to the geodesic parametrized by arc-length $\tilde{\gamma}_{1}$ defined by

$$
\tilde{\gamma}_{1}(t):=\gamma_{1}\left(\left(1+\kappa^{2}\right)^{-1 / 2} t\right)
$$

yields $\delta\left(\tilde{\gamma}_{1}(t)\right)>\kappa t$, whence

$$
\delta\left(\gamma_{1}(t)\right)>\left(1+\kappa^{2}\right)^{1 / 2} \kappa t>\kappa t .
$$

Consequently, for every $t \in(0, r]$, every geodesic defined by a unit vector $Y \in T_{\gamma_{1}(t)} M_{0}$ is defined at least up to the time $\kappa t$. Taking $Y$ to be the speed vector of the geodesic $s \rightarrow \exp _{\gamma(t)} s X$ at $s=\kappa t$, we obtain that this geodesic can actually be extended for $s \in[0,2 \kappa t]$, for any $t \in(0, r]$. By Lemma 4.3 (ii), the curve $\gamma_{2}:(0, r] \rightarrow M_{0}$ defined by $\gamma_{2}(t):=\exp _{\gamma(t)} 2 \kappa t X$ is thus a geodesic in $M_{0}$ with $\left|\dot{\gamma}_{2}\right|^{2}=1+4 \kappa^{2}$. Again, we check that the distance from $\gamma_{2}(t)$ to the singularity is at least $\kappa t$, showing that for every $t \in(0, r], \exp _{\gamma(t)} s X$ is well-defined for $|s| \leq 3 \kappa t$. Iterating the same argument shows that the geodesic $\exp _{\gamma(t)} s X$ is actually defined for every $t \in(0, r]$ and for every $s \in \mathbb{R}$

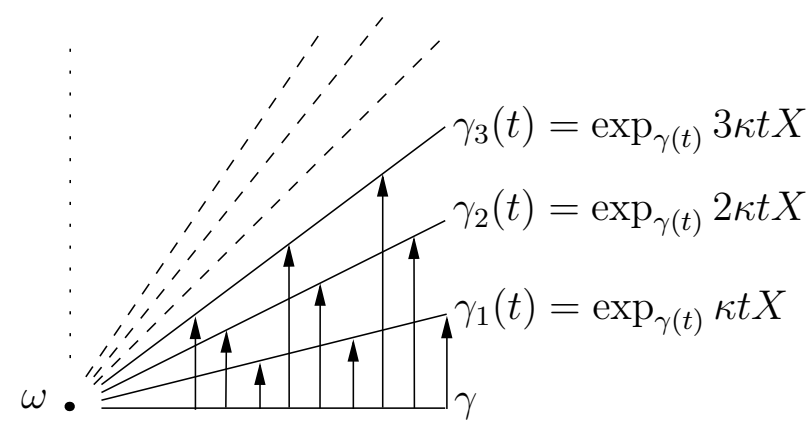

FiguRE 3. Idea of the proof of Proposition 4.5.

In particular, for $t=r, \gamma(t)=x$, we have proved that the geodesic through $x$ tangent to $X \in V_{2}$ is complete. Since $X$ was arbitrarily chosen, the whole integral leaf of $V_{2}$ through $x$ is thus complete.

In order to apply this result, we need to show that incomplete leaves actually exist.

Lemma 4.6. There exist incomplete maximal leaves $M_{i}$ of $V_{i}$ or, equivalently, incomplete geodesics $\gamma_{i}$ tangent to $V_{i}$ for $i=1$ and $i=2$.

Proof. Let $\gamma:(0,1] \rightarrow M_{0}$ be an incomplete geodesic, such that $\lim _{t \rightarrow 0} \gamma(t)=\omega$ in $\left(\widehat{M}_{0}, d\right)$. We may assume that $\gamma$ is not tangent to $V_{1}$ or $V_{2}$ : If for instance $\gamma$ were tangent to $V_{1}$, we replace it by $\gamma^{X}$ given by Lemma 4.3 (ii), which is neither tangent to $V_{1}$ nor to $V_{2}$. 
Let $X_{1}$ and $X_{2}$ denote the projections of $\dot{\gamma}$ on $V_{1}$ and $V_{2}$ respectively, which are clearly parallel along $\gamma$. We denote by $r_{i}:=\left|X_{i}\right| \neq 0$ the norms of $X_{i}$ and by $r:=\sqrt{r_{1}^{2}+r_{2}^{2}}$ the norm of $\dot{\gamma}$. Define the slope of $\gamma$ to be the quotient $q(\gamma):=r_{1} / r_{2}$.

We claim that $\gamma_{1}(t):=\exp _{\gamma(t)}\left(-t X_{1}\right)$ is defined for every $t \in(0,1]$, and is an incomplete geodesic tangent to $V_{2}$, such that $\lim _{t \rightarrow 0} \gamma_{1}(t)=\omega$ in $\left(\widehat{M}_{0}, d\right)$. The argument is similar to that used in the proof of Proposition 4.5: The exponential, denoted $\gamma^{s}(t)$ of $-t s X_{1}$ at $\gamma(t)$ is well-defined by Proposition 3.3 for $|s| \leq \frac{\kappa r}{r_{1}}$. For every fixed $s$ in this interval, $\gamma^{s}(t)$ is an incomplete geodesic and its slope is (see Lemma 4.3 (ii)) $q\left(\gamma^{s}\right)=(1-s) q(\gamma)$. If $\frac{\kappa r}{r_{1}} \geq 1$, which is equivalent to $q(\gamma) \leq\left(\kappa^{-2}-1\right)^{-1 / 2}$, the incomplete geodesic $\gamma^{s}$ has zero slope for $s=1$, i.e. it is tangent to $V_{2}$. Otherwise, we replace $\gamma$ by $\gamma^{s}$ with $s=\frac{\kappa r}{r_{1}}$ and repeat this procedure. The slope of the new geodesic is

$$
q\left(\gamma^{s}\right)=\left(1-\frac{\kappa r}{r_{1}}\right) q(\gamma)=q(\gamma)-\frac{\kappa r}{r_{2}} \leq q(\gamma)-\kappa
$$

showing that the procedure stops after a finite number of iterations. Since $V_{1}$ and $V_{2}$ play symmetric rôles, this finishes the proof.

Corollary 4.7. If there exists an incomplete geodesic $\gamma$ passing through a point $x \in M_{0}$ such that $\dot{\gamma}$ is neither tangent to $V_{1}$ nor to $V_{2}$, then $x$ belongs to a complete leaf of $V_{1}$ which intersects an incomplete maximal leaf of $V_{2}$, and to a complete leaf of $V_{2}$ which intersects an incomplete maximal leaf of $V_{1}$.

Proof. The result follows directly from the proof of Lemma 4.6 together with Proposition 4.5.

Lemma 4.8. If $M_{1}$ is a maximal leaf of $V_{1}$ which is incomplete, then the universal coverings of all maximal leaves of $V_{2}$ which intersect $M_{1}$ are isometric.

Proof. Let $M_{2}(x)$ denote the maximal leaf of $V_{2}$ through $x$. Since every two points of $M_{1}$ can be joined by a broken geodesic, it is enough to show that $M_{2}(\gamma(0))$ and $M_{2}(\gamma(1))$ are locally isometric for every geodesic $\gamma:[0,1] \rightarrow M_{1}$. Let $y$ be any point in $M_{2}(\gamma(0))$ and $U_{2}$ a simply connected neighborhood of $y$ in $M_{2}(\gamma(0))$. Consider the normal vector field $X$ on $U_{2}$ obtained by parallel transport of $\dot{\gamma}(0)$. Since $M_{2}(\gamma(0))$ is complete, $y$ can be expressed as $y=\exp _{\gamma(0)}(Y)$ for some $Y \in T_{\gamma(0)} M_{2}(\gamma(0))$. We extend $Y$ along $\gamma$ by parallel transport. By Proposition 4.5, the leaves $M_{2}(\gamma(t))$ are complete, hence $\exp _{\gamma(t)}(s Y)$ is well-defined for every $s \in \mathbb{R}$ and $t \in[0,1]$. By Lemma 4.3 (i) we get $\exp _{\gamma(t)}(Y)=\exp _{y}(t X)$. The exponential of $t X$ is thus defined for all points of $U_{2}$ and $t \in[0,1]$ so Lemma 4.2 shows that $U_{2}$ is isometric with its image by $\exp (X)$ in $M_{2}(\gamma(1))$.

The very same argument actually shows that the globally defined vector field obtained by parallel transport on the universal covering of $M_{2}(\gamma(0))$ defines a map $\left.\widehat{M_{2}(\gamma(0)}\right) \rightarrow M_{2}(\gamma(1))$ which is a local isometry. This map lifts to a local isometry $\left.\left.\widehat{M_{2}(\gamma(0)}\right) \rightarrow \widehat{M_{2}(\gamma(1)}\right)$ which is a global isometry as these two manifolds are complete and simply connected.

Consider now a metric $g$ on $M_{0}$ obtained as the pull-back of a metric in the conformal class $c$ on $M$. Let $\varphi$ be the conformal factor relating $g$ to $g_{0}$ by $g_{0}=\varphi^{2} g$ and let $\tilde{d}$ the geodesic distance induced on $M_{0}$ by $g$. Denote by $B_{x}(r)$ and $\tilde{B}_{x}(r)$ the set of points at distance less than $r$ from $x$ with respect to $d$ and $\tilde{d}$ respectively. Recall that by Lemma $2.10 \varphi$ is quasi-linear, so there exist 
positive constants $k_{1}, k_{2}$ such that

$$
k_{1} \delta(x) \leq \varphi(x) \leq k_{2} \delta(x), \quad \forall x \in M_{0} .
$$

Lemma 4.9. For every $x \in M_{0}$ and positive real number $r$, the open ball $B_{x}(r)$ contains the open ball $\tilde{B}_{x}(\tilde{r})$, where $\tilde{r}=\frac{r}{k_{2}(\delta(x)+r)}$.

Proof. For every $y \in \overline{B_{x}(r)}$ we have $\delta(y) \leq \delta(x)+r$, so by $(4) \varphi(y) \leq k_{2}(\delta(x)+r)$. Consequently, the $g^{0}$-length $l^{0}(c)$ and $g$-length $l(c)$ of every path contained in $\overline{B_{x}(r)}$ are related by

$$
l^{0}(c) \leq k_{2}(\delta(x)+r) l(c) .
$$

Assume there exists $z \in \tilde{B}_{x}(\tilde{r}) \backslash B_{x}(r)$ and let $c:[0,1] \rightarrow M_{0}$ be any path joining $x$ and $z$. Define $s_{0}=\inf \left\{s \mid c(s) \notin B_{x}(r)\right\}$ and consider the path $c^{\prime}=\left.c\right|_{\left[0, s_{0}\right]}$ which is clearly contained in $\overline{B_{x}(r)}$. Then $l^{0}\left(c^{\prime}\right) \geq r$, so by $(5), l(c) \geq l\left(c^{\prime}\right) \geq \tilde{r}$. Since this holds for every path $c$, we must have $\tilde{d}(x, z) \geq \tilde{r}$, contradicting the fact that $z \in \tilde{B}_{x}(\tilde{r})$.

Lemma 4.10. Let $\gamma:(0, a] \rightarrow M_{0}$ be an incomplete $g_{0}$-geodesic parametrized by arc-length, such that $\lim _{t \rightarrow 0} \delta(\gamma(t))=0$. There exist positive real numbers $\rho, q \in(0,1)$ such that the open balls $B_{n}:=B_{\gamma\left(q^{n}\right)}\left(\rho q^{n}\right), n \in \mathbb{N}$, are all pairwise disjoint.

Proof. Recall that by Proposition 3.3 we have control on the distance from $\gamma(t)$ to the singularity $\omega$, i.e. there exists a constant $\kappa \in(0,1)$, independent of $\gamma$, such that:

$$
\kappa t \leq \delta(\gamma(t)) \leq t, \quad \forall t \in(0, a]
$$

We start with arbitrary $\rho$ and $q$ in $(0,1)$. For every $y \in B_{n}$, Equation (6) yields

$$
(\kappa-\rho) q^{n} \leq \delta\left(\gamma\left(q^{n}\right)\right)-\rho q^{n} \leq \delta(y) \leq \delta\left(\gamma\left(q^{n}\right)\right)+\rho q^{n} \leq(\rho+1) q^{n},
$$

so by (4) we get

$$
k_{1}(\kappa-\rho) q^{n} \leq \varphi(y) \leq k_{2}(\rho+1) q^{n} .
$$

It is thus enough to choose $\rho$ and $q$ such that $k_{2}(\rho+1) q^{n+1}<k_{1}(\kappa-\rho) q^{n}$ for every $n$, which is equivalent to $\rho<\kappa$ and $q<\frac{k_{1}}{k_{2}(\rho+1)}(\kappa-\rho)$.

Corollary 4.11. Consider the open subset $B:=\cup_{n \geq 1} B_{n}$ in $M_{0}$. There exists $f \in \Gamma$ different from the identity such that $f(B) \cap B \neq \emptyset$.

Proof. Lemma 4.9 applied to $x=\gamma\left(q^{n}\right)$ and $r=\rho q^{n}$ shows that $B_{n}$ contains the open ball $\tilde{B}_{x}(\tilde{r})$ where

$$
\tilde{r}=\frac{r}{k_{2}(\delta(x)+r)}=\frac{\rho q^{n}}{k_{2}\left(\delta(x)+\rho q^{n}\right)} \geq \frac{\rho q^{n}}{k_{2}\left(q^{n}+\rho q^{n}\right)}=\frac{\rho}{k_{2}(1+\rho)} .
$$

Recall that $\Gamma$ acts by isometries on $\left(M_{0}, g\right)$ and that $\left(M_{0}, g\right) / \Gamma=(M, g)$. If $f\left(B_{n}\right) \cap B_{m}=\emptyset$ for every $f \in \Gamma$ and $m \neq n$, the projections $\pi\left(B_{n}\right)$ of $B_{n}$ onto $M$ would be pairwise disjoint sets, each of them containing a ball of $g$-radius $\frac{\rho}{k_{2}(1+\rho)}$ in $M$. This is impossible since $M$ is compact, thus proving our assertion.

The last step in the proof of Theorem 4.1 is the following:

Lemma 4.12. Let $M_{1}$ be a maximal leaf of $V_{1}$ which is incomplete. Then for every $x \in M_{1}$, the maximal leaf $M_{2}(x)$ of $V_{2}$ through $x$ is flat. 
Proof. Let $\gamma:(0,1] \rightarrow M_{1}$ be an incomplete geodesic with respect to $g_{0}$ parametrized by arc-length, such that $\gamma(1)=x$ and $\gamma(t)$ converges to $\omega$ (with respect to $d$ ) as $t$ tends to 0. By Lemma 4.10 one can find $\rho, q \in(0,1)$ such that the open balls $B_{n}:=B_{\gamma\left(q^{n}\right)}\left(\rho q^{n}\right)$ are pairwise disjoint. Moreover, one can choose $\rho$ such that each maximal leaf of $V_{2}$ through a point of $B=\cup_{n \geq 1} B_{n}$ intersects $M_{1}$. Indeed, this follows from Lemma 4.4 provided that $\rho q^{n}$ is smaller than $\sigma\left(\gamma\left(q^{n}\right)\right)$ for every $n$. Since $\sigma$ is quasi-linear, there exists some $\sigma_{0}$ such that $\sigma(x) \geq \sigma_{0} \delta(x)$, so from (6) it suffices to take $\rho<\kappa \sigma_{0}$.

Corollary 4.11 now shows that there exists $f \in \Gamma$ different from the identity and $y, z \in B$ such that $y=f(z)$. Then $f$ maps the integral leaf $M_{2}(z)$ of $V_{2}$ through $z$ to the integral leaf $M_{2}(y)$ of $V_{2}$ through $y$. Since both leaves intersect $M_{1}$, Lemma 4.8 shows that there exists a global isometry between their universal coverings $\widehat{M_{2}(y)}$ and $\widehat{M_{2}(z)}$. Moreover, $f$ lifts to a strict homothety $\tilde{f}: \widetilde{M_{2}(z)} \rightarrow \widetilde{M_{2}(y)}$. Composing $\tilde{f}$ with the isometry above, we obtain a strict homothety of $\widetilde{M_{2}(z)}$. Since $M_{2}(z)$ is complete, Lemma 2, page 242 in [10] shows that it must be flat. By Lemma 4.8 again, all the other leaves tangent to $V_{2}$ must be flat as well.

We are now in position to complete the proof of Theorem 4.1. From Corollary 4.7, and Lemma 4.12 , the sectional curvature of $g_{0}$ vanishes at each point $x$ which belongs to an incomplete geodesic which is neither tangent to $V_{1}$ nor to $V_{2}$. The proof of Lemma 4.3 (ii) shows that the set of such points is dense in $M_{0}$. Thus $\left(M_{0}, g_{0}\right)$ is a flat Riemannian manifold, so the holonomy group of $D=\nabla^{0}$ is discrete.

Remark 4.13. The only place where the compactness assumption on $M$ is needed in Theorem 4.1 , is to ensure, by Theorem 1.5, that the minimal Riemannian cover of $(M, c, D)$ has exactly one singularity. Theorem 4.1 thus holds in a slightly more general setting, and applies in particular to all Riemannian cones over complete Riemannian manifolds.

\section{Stable Weyl COnNeCtions}

In this section we introduce the notion of stability for Weyl connections and show that a stable Weyl connection is necessarily tame. As stability is an open condition in the $C^{1}$-topology, this shows, in particular, that the class of tame closed Weyl connections is significantly large.

Definition 5.1. A Weyl connection $D$ on a conformal manifold $(M, c)$ is called stable if there exists a complete Riemannian metric $g \in c$ and a positive real number $\varepsilon>0$ such that

$$
|\theta|^{2} g(X, X)+\left(\nabla_{X} \theta\right)(X) \geq 2 \varepsilon g(X, X), \quad \forall X \in T M,
$$

where $\nabla$ denotes the Levi-Civita covariant derivative of $g$ and $\theta$ denotes the Lee form of $D$ with respect to $g$.

Recall [8] that the Lee form $\theta$ of a Weyl connection $D$ with respect to a metric $g \in c$ measures the difference between $D$ and the Levi-Civita connection $\nabla=\nabla^{g}$ of $g$ :

$$
D_{X} Y-\nabla_{X} Y=\tilde{\theta}_{X}(Y):=\theta(X) Y+\theta(Y) X+\theta^{\sharp} g(X, Y), \forall X, Y \in T M,
$$

where $\theta=g\left(\theta^{\sharp}, \cdot\right)$.

Remark 5.2. With the notations from Example 2.5, it is easy to see that the standard Weyl connection $D_{0}$ on (a compact quotient of) a metric cone is stable. Indeed, the Lee form of $D_{0}$ with 
respect to the complete metric $g$ is $\theta_{0}:=d s$ and it is parallel for $\nabla=\nabla^{g}$. Therefore $\left|\theta_{0}\right|^{2} g+\nabla \theta=g$ thus ( 7 ) is even an equality for $\varepsilon=1 / 2$.

Remark 5.3. An exact Weyl connection on a compact conformal manifold $M$ can not be stable. Indeed, its Lee form $\theta$ with respect to any metric is exact, $\theta=d \varphi$ so (7) cannot hold at points where $\varphi$ reaches its maximum on $M$.

The remaining part of this section is devoted to the:

Proof of Theorem 1.6. Let $\theta$ be the Lee form of $D$ with respect to $g$. If $\nabla$ denotes the Levi-Civita covariant derivative of $g$, then we get from (8), see also [8]:

$$
D_{X}-\nabla_{X}=(\theta \wedge X)_{*}-p \theta(X) \operatorname{Id}
$$

on $T^{*} M^{\otimes p}$, where $(\theta \wedge X)_{*}$ is the usual extension of the endomorphism $\theta \wedge X$ as a derivation (in fact, the right hand side of (9) is just $\tilde{\theta}_{X}$, acting as a derivation on $\left.T^{*} M^{\otimes p}\right)$. It is easy to check that $(\theta \wedge X)_{*} g=0$, so (9) yields

$$
D_{X} g=-2 \theta(X) g .
$$

On the other hand, applying (9) to the Lee form $\theta$ itself yields

$$
D_{X} \theta=\nabla_{X} \theta+g(\theta, \theta) g(X, .)-2 \theta(X) \theta
$$

thus showing that (7) is equivalent to

$$
\left(D_{X} \theta\right)(X) \geq 2 \varepsilon g(X, X)-2 \theta(X)^{2}, \quad \forall X \in T M .
$$

Let $\gamma(t)$ be a geodesic with respect to $D$ on $M$ and let $I=(a, b)$ denote its maximal domain of definition, with $a, b \in \overline{\mathbb{R}}=\mathbb{R} \cup\{ \pm \infty\}$. We introduce the "speed" and "slope" functions $F(t):=$ $g(\dot{\gamma}(t), \dot{\gamma}(t))^{-\frac{1}{2}}$ and $H(t):=\theta(\dot{\gamma}(t))$, defined on $I$. Using (10) we get

$$
F^{\prime}(t)=\dot{\gamma}(t) \cdot F(t)=-\frac{1}{2}\left[\left(D_{\dot{\gamma}(t)} g\right)(\dot{\gamma}(t), \dot{\gamma}(t))\right]\left[g(\dot{\gamma}(t), \dot{\gamma}(t))^{-\frac{3}{2}}\right]=F(t) H(t),
$$

and from (11),

$$
H^{\prime}(t)=\dot{\gamma}(t) \cdot H(t)=\left(D_{\dot{\gamma}(t)} \theta\right)(\dot{\gamma}(t)) \geq 2 \varepsilon F(t)^{-2}-2 H(t)^{2} .
$$

Lemma 5.4. If $b<\infty$ (i.e. $\gamma$ is incomplete toward the future) then $\underline{\lim }_{t \rightarrow b} F(t)=0$. Similarly, if $a>-\infty$, then $\underline{\lim }_{t \rightarrow a} F(t)=0$.

Proof. We have to show that the $g$-norm of the speed vector of an incomplete $D$-geodesic cannot be bounded on $M$. Consider the geodesic flow of $D$, viewed as a vector field on the tangent bundle $T M$. Let $\gamma$ be the maximal half-geodesic with respect to $D$, issued from some $X \in T_{x} M$. There exists $T>0$ such that the maximal integral curve through $X$ of the geodesic flow is defined only for $t<T$. Assume that the $g$-norm of $\dot{\gamma}$ is bounded: $g(\dot{\gamma}(t), \dot{\gamma}(t))<k^{2}$ for all $t \in[0, T)$. Then the corresponding integral curve is contained in the subset $K(k, T)$ of $T M$ defined by

$$
K(k, T):=\left\{Y_{y} \in T M \mid d(x, y) \leq k T \text { and } g(Y, Y) \leq k^{2}\right\}
$$

where $d$ denotes the geodesic distance with respect to $g$. Since $g$ is complete, the closed geodesic balls are compact, so $K(k, T)$ is a compact subset of $T M$. On the other hand, it is well-known that an incomplete integral curve of a vector field cannot be contained in any compact subset, thus proving the lemma. 
In order to fix the ideas, we will assume from now on that

$$
0 \in I \quad \text { and } \quad H(0) \leq 0
$$

(this can always be achieved by making a translation in time and replacing $\gamma(t)$ with $\gamma(-t)$ if necessary).

Lemma 5.5. The function $F$ has at most one critical point. If this happens, then the critical point is an absolute minimum of $F$, and $\gamma$ is complete. Conversely, if $\gamma$ is complete, then $F$ has a critical point.

Proof. Let $t_{0}$ be a critical point of $F$. From (12), $H\left(t_{0}\right)=0$. Moreover, (13) shows that $H^{\prime}$ is strictly positive at each point where $H$ vanishes, so actually $H$ cannot vanish more than once. Thus $H(t)$ is positive for $t \geq t_{0}$ and negative for $t \leq t_{0}$, so $t_{0}$ is a global minimum of $F$. Lemma 5.4 then shows that $\gamma$ cannot be incomplete.

Conversely, assume that $\gamma$ is complete, i.e. $I=\mathbb{R}$. If $F$ has no critical point, $H$ does not vanish, so by our assumption (14), $H<0$ on $\mathbb{R}$. $F$ is thus a decreasing positive function, so $\lim _{t \rightarrow \infty} H(t) F(t)=\lim _{t \rightarrow \infty} F^{\prime}(t)=0$. Dividing by $H(t)^{2}$ in (13) yields

$$
\frac{H^{\prime}(t)}{H(t)^{2}}>\frac{2 \varepsilon}{F(t)^{2} H(t)^{2}}-2 \text {. }
$$

Since the right hand side tends to infinity as $t \rightarrow \infty$, an integration shows that $\lim _{t \rightarrow \infty} H(t)=0$. Using (13) again, we then see that there exist some $t_{0} \in \mathbb{R}$ and $\delta>0$ such that $H^{\prime}(t)>\delta$ for $t>t_{0}$. This of course contradicts the fact that $H$ is negative on the whole real line $\mathbb{R}$, thus proving the lemma.

The convention (14) together with Lemma 5.5 ensures that if $\gamma$ is an incomplete geodesic, $H$ is negative on $I$, so $F$ is decreasing. By Lemma 5.4, $\gamma$ is complete toward $-\infty$, i.e. $I=(-\infty, b)$, with $b \in \mathbb{R}_{+}$.

Lemma 5.6. If $\gamma: I \rightarrow M_{0}$ is a geodesic with respect to $D$ which is incomplete in the positive direction, then

$$
H(t) \leq-\frac{\sqrt{\varepsilon}}{F(t)}, \quad \forall t \in I
$$

Proof. If (15) does not hold, there exists $t_{0} \in I$ such that

$$
-\frac{\sqrt{\varepsilon}}{F\left(t_{0}\right)}<H\left(t_{0}\right)<0
$$

We define the open set

$$
I^{\prime}:=\left\{t \in I \mid-\frac{\sqrt{\varepsilon}}{F(t)}<H(t)\right\},
$$

and let $\left(a^{\prime}, b^{\prime}\right)$ be the connected component of $I^{\prime}$ containing $t_{0}$. By (13), $H$ is strictly increasing on $I^{\prime}$. In the other hand, we have seen that $F$ is decreasing on $I$. If $b^{\prime} \in I$, we would have

$$
H\left(b^{\prime}\right)=-\frac{\sqrt{\varepsilon}}{F\left(b^{\prime}\right)}<-\frac{\sqrt{\varepsilon}}{F\left(t_{0}\right)}<H\left(t_{0}\right),
$$


a contradiction. The only possibility left is thus $b^{\prime}=b$. But this is impossible as well, since by $(12)$,

$$
\lim _{t \rightarrow b^{\prime}} \log (F(t))=\log \left(F\left(t_{0}\right)\right)+\int_{t_{0}}^{b^{\prime}} H(t) d t>-\infty,
$$

contradicting Lemma 5.4.

Back to the proof of Theorem 1.6, using (15) and (12) we get $F^{\prime}(t) \leq-\sqrt{\varepsilon}$, and thus

$$
\sqrt{\varepsilon} b=\int_{0}^{b} \sqrt{\varepsilon} d t \leq-\int_{0}^{b} F^{\prime}(t) d t \leq F(0)=g(\dot{\gamma}(0), \dot{\gamma}(0))^{-\frac{1}{2}} .
$$

In other words, the life-time of every geodesic $\gamma$, incomplete in the positive direction, is bounded from above by $(\varepsilon g(\dot{\gamma}(0), \dot{\gamma}(0)))^{-\frac{1}{2}}$. Let $K$ be any compact subset of $T M \backslash\{0\}$ and let $l(K)$ denote

$$
l(K):=\inf _{X \in K}\{g(X, X)\} .
$$

With the notations from Section 3, for every $X \in \mathcal{I}^{D} \cap K$ we have $\mathcal{L}^{D}(X) \leq(\varepsilon l(K))^{-\frac{1}{2}}$, so $D$ is tame by Proposition 3.3.

The stability condition (7) is clearly open in the $C^{1}$ topology defined by the metric $g$ on the space of Weyl connections. Therefore, Theorem 4.1 applies to open subsets of the space of closed Weyl connections.

Example 5.7. Let $h_{t}$ be a $T$-periodic 1-parameter family of metrics on a compact manifold $N$ and let $g:=d t^{2}+h_{t}$ be the generalized cylinder metric defined on $M:=\mathbb{R} \times N$, with Levi-Civita connection $\nabla$. It is straightforward to check that

$$
\nabla_{\frac{\partial}{\partial t}} \frac{\partial}{\partial t}=0, \quad\left[\frac{\partial}{\partial t}, Y\right]=0, \quad g\left(\nabla_{Y} \frac{\partial}{\partial t}, Y\right)=g\left(\nabla_{\frac{\partial}{\partial t}} Y, Y\right)=\frac{1}{2} \dot{h}_{t}(Y, Y), \quad \forall Y \in T N .
$$

By compactness, there exists some positive real number $s_{0}$ such that $s_{0} h_{t}+\frac{1}{2} \dot{h}_{t}$ is positive definite for all $t$. Then for every $s>s_{0}$, the Weyl connection $D^{s}$ whose Lee form with respect to $g$ is $\theta^{s}:=s d t$, is stable. Indeed, for any $X \in T M$ written as $X=a \frac{\partial}{\partial t}+Y$ with $Y \in T N$, we can express the left hand term of Inequality (7) using (16) as

$$
|\theta|^{2} g(X, X)+\left(\nabla_{X} \theta\right)(X)=s^{2}\left(a^{2}+h_{t}(Y, Y)\right)+\frac{1}{2} s \dot{h}_{t}(Y, Y),
$$

therefore (7) is satisfied for $2 \varepsilon:=\min \left\{s_{0}^{2}, s^{2}-s s_{0}\right\}$. If $\Gamma$ denotes the group generated by the $g$-isometry $(t, x) \mapsto(t+T, x)$, then $D^{s}$ defines a closed, non-exact, stable Weyl connection on the compact manifold $M / \Gamma$. Moreover, for $\dot{h}_{t}$ large with respect to $h_{t}$, this connection is not $C^{1}$-close to a quotient of a cone (which corresponds to the case $\dot{h}_{t} \equiv 0$ ).

\section{EXAMPLES AND APPLICATIONS}

6.1. Holonomy issues. An exact Weyl connection on a conformal manifold is just the Levi-Civita connection of some metric in the conformal class. The possible restricted holonomy groups of exact Weyl connections are thus given by the Berger-Simons theorem ([4], p. 300). The analogous question for non-closed Weyl connections can be answered from [12] in the irreducible case and was studied in [3] in the reducible case. It thus remains to understand the case of closed, non-exact Weyl connections. The next result - which gives a complete list in the compact case, under the 
assumption that the connection is tame - is a direct consequence of Theorem 4.1 and well known facts.

Theorem 6.1. The restricted holonomy group of a closed, non-exact, tame Weyl connection $D$ on a compact $n$-dimensional conformal manifold $(M, c)$ is one of the following:

$$
\left.\left.\mathrm{SO}(n), \mathrm{U}(n / 2), \mathrm{SU}(n / 2), \mathrm{Sp}(n / 4), \mathrm{G}_{2} \text { (for } n=7\right) \text {, } \mathrm{Spin}(7) \text { (for } n=8\right), 0 .
$$

Conversely, each of the groups listed above can be realized as the restricted holonomy of a closed, non-exact Weyl connection on a compact conformal manifold.

Proof. Since $D$ is locally the Levi-Civita connection of metrics in the conformal class $c$, the BergerSimons theorem applies. Assume first that $D$ is locally symmetric. The metric $g_{0}$ on the minimal Riemannian cover $M_{0}$ of $(M, c, D)$ is then locally symmetric. Every nontrivial homothety $f$ satisfies $f^{*} g_{0}=\rho(f)^{2} g_{0}$ and preserves the Riemannian curvature tensor $R_{0}$. In particular $f^{*}\left(\left|R_{0}\right|^{2}\right)=$ $\rho(f)^{-4}\left|R_{0}\right|^{2}$. On the other hand, $R_{0}$ being parallel with respect to the Levi-Civita connection of $g_{0},\left|R_{0}\right|^{2}$ is constant on $M_{0}$. Since $\rho(f) \neq 1$, this shows that $\left(M_{0}, g_{0}\right)$ is flat, $\operatorname{so} \operatorname{Hol}_{0}(D)=0$.

Assuming from now on that $\operatorname{Hol}_{0}(D) \neq 0, D$ is irreducible by Theorem 4.1, $\operatorname{so}^{H_{0}}(D)$ is in the Berger list [4], p. 301. It remains to show that $\mathrm{Sp}(k) \cdot \mathrm{Sp}(1)$ can not be realized as the restricted holonomy group of a closed, non-exact Weyl connection. The argument is similar to the one used above. If $\mathrm{Hol}_{0}(D)=\mathrm{Sp}(k) \cdot \mathrm{Sp}(1)$ then the minimal cover $\left(M_{0}, g_{0}\right)$ is quaternion-Kähler, therefore Einstein with non-zero Ricci tensor Ric $=\lambda g_{0}$ [4]. Since the homotheties that act on $M_{0}$ preserve the Levi-Civita connection of $g_{0}$, they also preserve the Ricci tensor. We infer that every homothety has to be an isometry, which contradicts the fact that $D$ is not exact.

Conversely, every group in the above list can be realized as the holonomy of a closed, non-exact Weyl connection on a compact manifold $M=S^{1} \times N$, obtained as quotient of the Riemannian cone over a manifold $(N, g)$ endowed with special structure by a non-trivial homothety (see [1] for details).

Note that the case $\operatorname{Hol}_{0}(M, D)=\mathrm{U}(m)$ is well-known in the literature and corresponds to locally conformally Kähler (l.c.K.) manifolds. The l.c.K. structure constructed above on $S^{1} \times N$ for every Sasakian manifold $N$ has the following special property: There exists a metric $g$ in the conformal class such that the Lee form of $D$ with respect to $g$ is $\nabla^{g}$-parallel [15]. This special kind of l.c.K. metric is called Vaisman metric and it is known that not every l.c.K. structure contains such a metric in the conformal class, not even for a deformation of the l.c.K. conformal class (see [11], [14] for examples of l.c.K. manifolds which can not be conformally Vaisman for topological reasons, having non-zero Euler characteristic, and also [2] for a classification of Vaisman structures on compact 4-manifolds).

For the other holonomy groups in the above list we have the following structure result (note that the tameness assumption is no longer required):

Theorem 6.2. Let $(M, c, D)$ be a compact Weyl manifold of dimension $n>2$, such that $D$ is a closed non-exact Weyl connection whose restricted holonomy is one of the following subgroups of $\mathrm{SO}(n): \mathrm{SU}(n / 2), \mathrm{Sp}(n / 4), \mathrm{G}_{2} \subset \mathrm{SO}(7), \mathrm{Spin}(7) \subset \mathrm{SO}(8)$ or $0 \subset \mathrm{SO}(n)$. Then the following hold:

(1) The minimal Riemannian cover of $(M, c, D)$ is a Riemannian cone.

(2) The manifold $M$, endowed with its Gauduchon metric, is a mapping torus. 
Proof. Let $g \in c$ denote the Gauduchon metric of $D$ on $M$ (which is determined up to a multiplicative constant by the fact that the Lee form of $D$ with respect to $g$ is $\delta^{g}$-co-closed, see [7]), as well as its pull-back to the universal cover $\tilde{M}$ of $M$. We denote by $g_{0}$ the metric on $\tilde{M}$ having $D$ as Levi-Civita covariant derivative. In all five cases (2)-(6), the metric $g_{0}$ is Ricci-flat, so $D$ is an Einstein-Weyl connection. This also holds on the compact manifold $M$, therefore Theorem 3 in [8] implies that the Lee form of $D$ with respect to $g$ is parallel (and non-zero). The same is true on the complete, simply connected manifold $(\tilde{M}, g)$, which is therefore isometric to a Riemannian product $\left(\mathbb{R}, d s^{2}\right) \times\left(N, g_{N}\right)$. The Lee form of $D$ with respect to $g$ on $\tilde{M}$ is just $d s$, so $g_{0}=e^{2 s} g$, i.e. $g_{0}=d t^{2}+t^{2} g_{N}$ after a coordinate change $t:=e^{s}$. This means that $\left(\tilde{M}, g_{0}\right)$ is the Riemannian cone over $\left(N, g_{N}\right)$. It is well-known, see for example [1], that if the holonomy of the Riemannian cone of $\left(N, g_{N}\right)$ is one of the five groups above, then $\left(N, g_{N}\right)$ is Einstein with positive scalar curvature. This, together with the fact that $N$ is closed in $\tilde{M}$, (and thus complete), implies that $N$ has to be compact.

Let $f \in \pi_{1}(M)$ be any deck transformation, thus acting isometrically on $(\tilde{M}, g)$. Since $f$ is affine with respect to $D$, it has to preserve the Lee form of $D$ with respect to g, i.e. $f^{*}(d s)=d s$, and therefore it preserves its $g$-dual $\partial / \partial s$. This means that $f$ commutes with the flow of $\partial / \partial s$ on $\tilde{M}$, so it is induced by an isometry, also denoted by $f$, of $\left(N, g_{N}\right): f(s, x)=(s+\ln (\rho(f)), f(x))$ (recall that $\rho(f)$ is the homothety constant of $f$ with respect to $\left.g_{0}: f^{*} g_{0}=\rho(f)^{2} g_{0}\right)$. It follows that the group $\mathcal{I} \subset \pi_{1}(M)$ of deck transformations preserving $g_{0}$ induces a group of isometries $\mathcal{I}_{N}$ acting freely on $\left(N, g_{N}\right)$, so the minimal Riemannian cover $\left(M_{0}, g_{0}\right)$ of $(M, c, D)$ is the Riemannian cone over $\left(N, g_{N}\right) / \mathcal{I}_{N}$.

Finally, the compactness of $N$ implies that the deck transformation group $\Gamma=\pi_{1}(M) / \mathcal{I}$ of the covering $M_{0} \rightarrow M$ is discrete, hence isomorphic to $\mathbb{Z}$, showing that $(M, g)$ is the mapping torus of an isometry of $\left(N, g_{N}\right) / \mathcal{I}_{N}$.

As a consequence, $\chi(M)=0$ and the fundamental group of $M$ is a finite extension of $\mathbb{Z}$. Note that if $\operatorname{dim} M=2$ and $D$ is flat, its minimal covering may be $\mathbb{C}^{*}$ or $\mathbb{C}$. In both cases $\pi_{1}(M)$ is (a finite extension of) $\mathbb{Z}^{2}$.

Remark 6.3. The Berger-Simons theorem, along with the de Rham decomposition theorem, completely classify the restricted holonomy groups of torsion-free connections with bounded full holonomy group (as a subset of $\operatorname{GL}(n, \mathbb{R}) \subset \mathbb{R}^{n^{2}}$ ). On the other hand, a closed, non-exact Weyl connection is just a torsion-free connection whose restricted holonomy group is compact, but its full holonomy group is not bounded. Theorem 4.1 and the results in this section can thus be interpreted as an holonomy classification for this kind of connections (under the tameness assumption).

6.2. An example of cone-like manifold which is not tame. Let $\widehat{C_{0}}$ be the following rotation cone in $\mathbb{R}^{3}$ :

$$
\widehat{C_{0}}:=\left\{(x, y, z) \mid z=\sqrt{x^{2}+y^{2}}\right\} .
$$

The set $C_{0}:=\widehat{C_{0}} \backslash\{0\}$ is a smooth Riemannian submanifold of $\mathbb{R}^{3}$ and its metric completion is $\widehat{C_{0}}$. The homothety $X \mapsto 2 X$ in $\mathbb{R}^{3}$ defines by restriction a homothety $f$ of $C_{0}$, which generates a group of homotheties $\Gamma:=\left\{f^{n} \mid n \in \mathbb{Z}\right\}$ acting freely and properly discontinuously on $C_{0}$. The quotient space is a topological torus $T^{2}$. The Riemannian metric on $C_{0}$ defines by projection a conformal 
structure on $T^{2}$, and its Levi-Civita connection projects to a closed, non-exact Weyl connection on $T^{2}$.

We are going to apply some surgery and smoothening to get by similar methods a closed, nonexact Weyl connection on a surface of genus 2 .

To do that, consider the domain $B_{0}:=C_{0} \cap\{1<z<2\}$, remove the two topological discs obtained as intersection of $B_{0}$ with the full cylinder

$$
Z_{0}:=\left\{(x, y, z) \in \mathbb{R}^{3} \mid y^{2}+(z-3 / 2)^{2} \leq 1 / 16\right\}
$$

connect the borders of the two removed discs by the part of the boundary of $Z_{0}$ that lies inside the cone $C_{0}$, then smoothen it up to get a new surface $B \subset \mathbb{R}^{3}$ such that:

(1) Only the part of $B_{0}$ inside the (larger) cylinder

$$
Z:=\left\{(x, y, z) \in \mathbb{R}^{3} \mid y^{2}+(z-3 / 2)^{2} \leq 1 / 8\right\}
$$

has been changed (in particular there are neighborhoods of the two boundary circles of $B_{0}$ that are unchanged, so the gluing with the remaining part of $C_{0}$ can be done smoothly);

(2) The symmetries

$$
S^{x}, S^{y}: \mathbb{R}^{3} \rightarrow \mathbb{R}^{3}, S^{x}(x, y, z):=(-x, y, z), S^{y}(x, y, z):=(x,-y, z)
$$

still act as isometries of $B$.

The union

$$
N_{0}:=\bigcup_{n \in \mathbb{Z}} f^{n}(\bar{B})
$$

is then a non-closed (hence incomplete) smooth submanifold in $\mathbb{R}^{3}$ which can be completed as a metric space by adding the origin to it. Let $g_{0}$ denote the induced Riemannian metric from $\mathbb{R}^{3}$. The group $\Gamma$ acts on $\left(N_{0}, g_{0}\right)$ by homotheties and the quotient space $N:=N_{0} / \Gamma$ is a genus 2 surface (obtained by gluing together the two circles that constitute the boundary of $B$ ). The Riemannian metric $g_{0}$ and its Levi-Civita connection define, by projection, a conformal structure $c$, and a closed, non-exact Weyl connection $D$ on $N$.
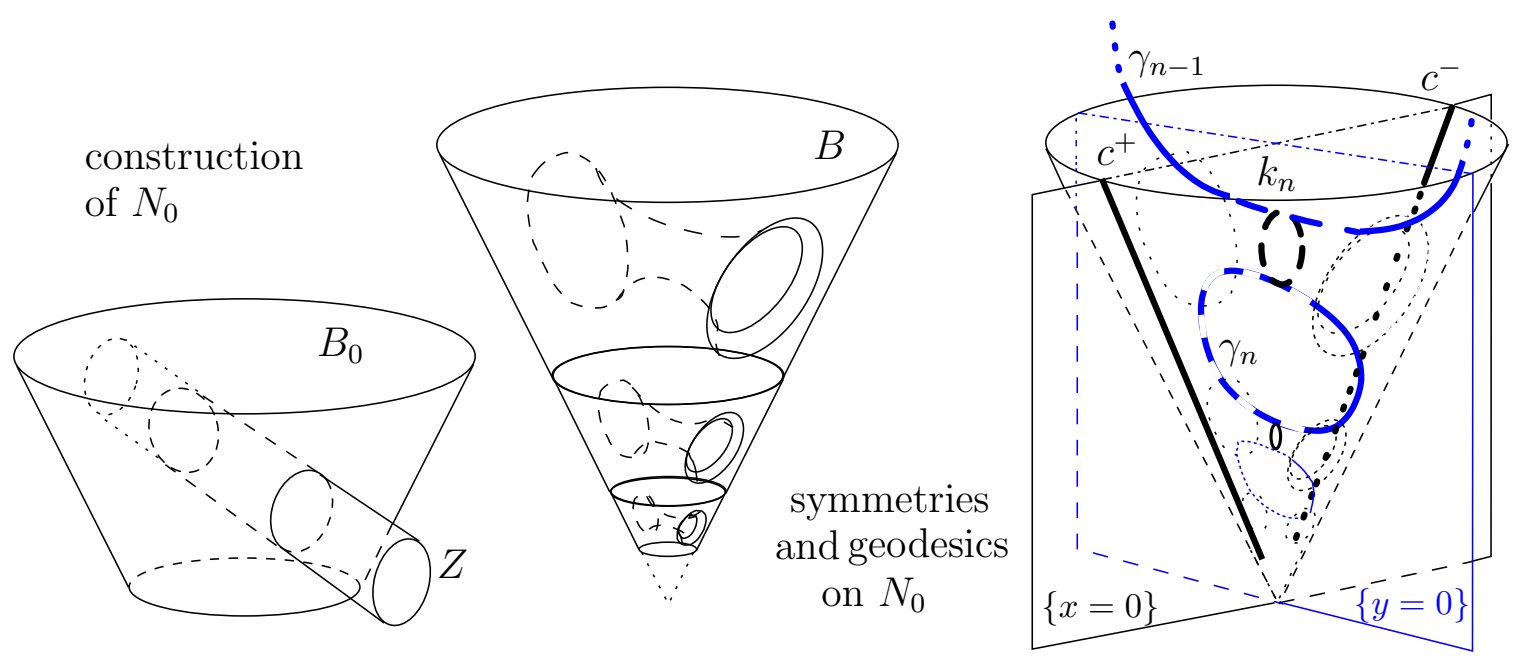

Figure 4. Construction and properties of $N_{0}$. 
We are going to study the geodesics of $N_{0}$ and prove the following

Proposition 6.4. The Weyl connection $D$ is not tame on $(N, c)$.

Proof. Consider the isometries $S^{x}$ and $S^{y}$ acting on $\left(N_{0}, g_{0}\right)$, whose fixed point sets consist of unions of geodesics in $N_{0}$ :

(1) $\operatorname{Fix}\left(S^{x}\right)=N_{0} \cap\{x=0\}$, which is a union of two half lines $c^{+}, c^{-}:(0, \infty) \rightarrow N_{0}, c^{ \pm}(t):=$ $(0, \pm t, t)$ and an infinity of circles $k_{n}:=\left\{(0, y, z) \mid y^{2}+\left(z-3 \cdot 2^{n-1}\right)^{2}=4^{n-2}\right\}, n \in \mathbb{Z}$;

(2) $\operatorname{Fix}\left(S^{y}\right)=N_{0} \cap\{y=0\}$, which is a union of closed curves $\gamma_{n}$ connecting $f^{n}(B)$ with $f^{n+1}(B)$ and intersecting $k_{n}$ in $P_{n}:=\left(0,0,7 \cdot 2^{n-2}\right)$, and $k_{n+1}$ in $Q_{n+1}:=\left(0,0,3 \cdot 2^{n-1}\right)$.

We denote $P:=P_{n}$, for some positive integer $n$. The point $P$ is a fixed point for both isometries $S^{x}$ and $S^{y}$, and hence for their composition $S:=S^{x} \circ S^{y}$. The latter induces the map $X \mapsto-X$ on $T_{P} N_{0}$ and associates to a point $Q \in N_{0}$ the geodesic reflection through $P$, i.e. the point $\bar{Q}$ such that, for any geodesic $c^{Q}:(-\varepsilon, a] \rightarrow N_{0}$ with $c^{Q}(a)=Q$ and $c^{Q}(0)=P, c^{Q}$ can be defined on a symmetric interval $[-a, a]$, and $\bar{Q}=c^{Q}(-a)$.

On the other hand, there exists a geodesic $\gamma:(-\varepsilon, T) \rightarrow N_{0}$ such that $\gamma(0)=P$ and $\gamma(t)$ tends to $\omega=(0,0,0)$ as $t$ tends to $T$. The remark above implies that the geodesic is actually defined on $(-T, T)$ (and this is its maximal domain of definition), and

$$
\lim _{t \rightarrow T} \gamma(t)=\omega=\lim _{t \rightarrow-T} \gamma(t),
$$

so both ends of the incomplete geodesic $\gamma$ tend to the singularity.

For any $\varepsilon>0$, the point $\gamma(T-\varepsilon)$ can thus be connected by at least two half-geodesics with $\omega$, namely the two branches of $\gamma$, of lengths $\varepsilon$ and $2 T-\varepsilon$ respectively. As $\varepsilon$ can be chosen arbitrarily small, we see that there is no bound for the ratios of those lengths, therefore $N_{0}$ is not tame by the converse statement in Proposition 3.3.

6.3. Reducible, non-conformal, locally metric connections. We give here an example of a non-conformal locally metric connection with reducible holonomy which is not flat and not globally a product.

Example 6.5. Let $(\tilde{M}, \tilde{g}):=\left(\tilde{M}_{1}, \tilde{g}_{1}\right) \times\left(\tilde{M}_{2}, \tilde{g}_{2}\right)$, where

$$
\left(\tilde{M}_{1}, \tilde{g}_{1}\right):=\left(\mathbb{R}_{+}^{*} \times S^{n}, d r^{2}+r^{2} g_{1}\right)
$$

is the Riemannian cone over a sphere endowed with a non-round metric $g_{1}$, and $\left(\tilde{M}_{2}, \tilde{g}_{2}\right):=\left(\mathbb{R}, d t^{2}\right)$ is just a line. We define now

$$
\gamma_{j}(r, x, t):=\left(e^{a_{j}} r, x, t+j a_{j}\right), j=1,2,
$$

where $a_{1}$ and $a_{2}$ are real numbers such that $a_{1} . a_{2}>0$ and $a_{1} / a_{2} \notin \mathbb{Q}$. We see that $\gamma_{j}$ act by affine transformations of $(\tilde{M}, \tilde{g})$. On the other hand, the group $\Gamma$, generated by $\gamma_{1}$ and $\gamma_{2}$ is Abelian and acts freely on $\tilde{M}$. One can also check that this action is proper, thus $M:=\tilde{M} / \Gamma$ is a manifold that inherits the Levi-Civita connection $D$ of $\tilde{g}$ but $D$ does not preserve any Riemannian metric on $M$. Moreover, the $D$-stable distributions (note that $\left(\tilde{M}_{1}, \tilde{g}_{1}\right)$ is an irreducible Riemannian manifold, because $g_{1}$ is not the round metric) on $M$ generate transversal foliations, but no global product structure. 
6.4. Open problems. Several natural questions emerge from the considerations above.

(1) Theorem 1.5 shows that if $D$ is a closed Weyl connection on a compact conformal manifold $(M, c)$, then the minimal Riemannian cover $\left(M_{0}, g_{0}\right)$ can be metrically completed by adding exactly one point. The metric completion of its universal covering $(\tilde{M}, \tilde{g})$ is, however, not well understood: The boundary of $\tilde{M}$ in its metric completion may be more complicated in general, possibly depending on the growth of the fundamental group of $M$.

(2) One can check that the stability condition forces the Lee form $\theta$ to be non-vanishing, therefore restricting the topology of $M_{0}$ to products $\mathbb{R} \times N$, in particular $\chi(M)=0$. Does the tameness condition also imply a topological restriction? And if this restriction is satisfied, is the connection automatically tame?

(3) Ultimately, does Theorem 4.1 hold without the tameness assumption?

A positive answer to this last question would be equivalent to Conjecture 1.3. Note that the only crucial place where tameness is used is Proposition 4.5, which ensures the existence of sufficiently many complete leaves on a reducible cone-like manifold.

\section{REFERENCES}

[1] C. B̈̈r, Real Killing spinors and holonomy, Commun. Math. Phys. 154 (1993), 509-521.

[2] F. Belgun, On the structure of non-Kähler complex surfaces, Math. Ann. 317 (2000), 1-40.

[3] F. Belgun, A. Moroianu, Weyl-parallel forms, conformal products and Einstein-Weyl manifolds, Asian J. Math. 15 (2011), 499-520.

[4] A. Besse, Einstein manifolds, Ergebnisse der Mathematik und ihrer Grenzgebiete (3) 10. Springer-Verlag, Berlin, 1987.

[5] Th. Friedrich, I. Kath, A. Moroianu, U. Semmelmann, On nearly-parallel G ${ }_{2}$-structures, J. Geom. Phys. 23 (1997), 269-286.

[6] S. Gallot, Équations différentielles caractéristiques de la sphère, Ann. Sci. Ec. Norm. Sup. Paris 12 (1979), 235-267.

[7] P. Gauduchon, La 1-forme de torsion d'une variété hermitienne compacte, Math. Ann. 267 (1984), $495-518$.

[8] P. Gauduchon, Structures de Weyl-Einstein, espaces de twisteurs et variétés de type $S^{1} \times S^{3}$, J. reine angew. Math. 469 (1995), 1-50.

[9] A. Gray, The structure of nearly Kähler manifolds, Math. Ann. 223 (1976), 233-248.

[10] S. Kobayashi, K. Nomizu, Foundations of Differential Geometry I, New York, Interscience Publishers, 1963.

[11] C. R. LeBrun, Anti-self-dual Hermitian metrics on blown-up Hopf surfaces, Math. Ann. 389 (1991), $383-392$.

[12] S. Merkulov, L. SchwachHöfer, Classification of irreducible holonomies of torsion-free affine connections, Ann. of Math. 150 (1999), 77-149.

[13] A. Moroinnu, L. Ornea, Conformally Einstein products and nearly Kähler manifolds, Ann. Global Anal. Geom. 33 (2008), 11-18.

[14] F. Tricceri, Some examples of locally conformal Kähler manifolds, Rend. Semin. Mat. Univ. Politecn. Torino 40 (1982), 81-92.

[15] I. VAISMAn, Locally conformal Kähler manifolds with parallel Lee form, Rend. Mat. (6) 12 (1979), $263-284$.

[16] H. WeYL, Raum. Zeit. Materie, (German), Heidelberger Taschenbücher, 251, Springer-Verlag, Berlin, 1988.

Florin Belgun, Fachbereich Mathematik, Bereich AD, Bundesstr. 55 (Geomatikum), 20146 HamBURG, GERMany

E-mail address: florin.belgun@math.uni-hamburg.de

Andrei Moroianu, Université de Versailles-St Quentin, Laboratoire de Mathématiques, UMR 8100 Du CNRS, 45 AVENUe des ÉtATs-Unis, 78035 Versailles, France

E-mail address: andrei.moroianu@math.cnrs.fr 